\title{
Vibrational Analyses of 2,4-Dichloropentane and 2,4,6-Trichloroheptane
}

\author{
W. H. MOORE AND S. KRIMM \\ Harrison $M$. Randall Laboratory of Physics, University of Michigan, \\ Ann Arbor, Michigan 48104
}

\begin{abstract}
This study of the vibrational spectra of 2,4-dichloropentane and 2,4,6-trichloroheptane uses normal coordinate calculations as a probe for the presence of different molecular conformers. The calculations use a complete valence force field for secondary chlorides which was developed by fitting the observed vibrational frequencies of model compounds. Observed and calculated frequencies agree well. The results of these calculations on $d l$ and meso 2,4-dichloropentane and on isotactic 2,4,6-trichloroheptane indicate the presence of conformers other than those which are accepted as sterically preferred.
\end{abstract}

\section{INTRODUCTION}

In order to elucidate the effect of stereochemical structure on conformation in poly(vinyl chloride) (PVC), the dimer and trimer models of PVC have been studied extensively (1-6). In these investigations nuclear magnetic resonance (NMR), infrared and Raman spectroscopy have been the most effective tools. In NMR, chemical shifts and coupling constants are found to be dircctly related to molccular configuration. However, the interpretation of NMR spectra is not based on a simple superposition of chemical shifts and coupling constants but on a weighted average of these parameters. This complicated averaging, which is a function of the total conformational population of the compound being studied, renders NMR spectroscopy less satisfactory for identifying rotational isomers of molecules which exist as a mixture of more than two conformers. Because infrared and Raman spectra comprise a simple superposition of contributions from all isomeric structures in equilibrium, complete conformer analysis by normal coordinate calculations is theoretically possible (although spectral overlap between various structures often renders the interpretation of the vibrational spectra difficult). In previous studies $(7,8)$ we have developed a complete general valence force field for secondary chlorides We present here the results of applying this force field to the analysis of the spectra of 2,4-dichloropentane and 2,4,6-trichloroheptane.

In this investigation of secondary chlorides we use a staggered molecular model, that is, one in which the dihedral angle associated with a given $\mathrm{C}-\mathrm{C}-\mathrm{C}-\mathrm{C}$ unit can only assume the discrete values of $-60^{\circ},+60^{\circ}$ or $+180^{\circ}$. (Departures from these specific values may, of course, occur in the stable conformations of the actual molecules.) Within the framework of this approximation small molecules can exist in a limited number of geometrically unique conformations, e.g., one conformation for 2-chloro- 
propane and three conformations for 2-chlorobutane. In such instances the interpretation of the vibrational spectra can be done almost uniquely by means of normal coordinate analyses. However, for each of the stereochemical isomers of 2,4-dichloropentane, six geometrically unique structures can be defined within the constraints of the staggered molecular model. Because of the overlap of the predicted absorption bands of these conformers, normal coordinate analyses alone cannot be used to determine the equilibrium conformer population of $d l$ - and meso-2,4-dichloropentane. Until recently many of the staggered conformational structures of 2,4-dichloropentane, and similar molecules in which large constituents such as chlorine atoms and methyl groups approach one another closely, have been considered to be sterically forbidden. This constraint, in conjunction with NMR and infrared investigations, led to the conclusion that at room temperature only two of the six possible conformers of $d l$-2,4-dichloropentane were present, the $T T$ (or ${ }_{\mathrm{H}} S_{\mathrm{HH}} S_{\mathrm{H}}$ ) and the $G G$ (or ${ }_{\mathrm{H}} S_{\mathrm{CC}} S_{\mathrm{H}}$ ) forms. In meso-2,4dichloropentane only the $T G^{\prime}\left({ }_{\mathrm{H}} S_{\mathrm{HC}} S_{\mathrm{H}}\right)$ conformation was believed to be present, in mobile equilibrium with its mirror image, $G^{\prime} T\left({ }_{\mathrm{H}} S_{\mathrm{CH}} S_{\mathrm{H}}\right)$. The dominant features of the infrared, Raman and NMR spectra of 2,4-dichloropentane are well explained by these major conformers. However, weak infrared bands in the highly diagnostic region of the carbon-chlorine stratching vibrations $(9)$, relatively strong and characteristic far infrared bands (7), and the temperature behavior of NMR spectra (4) cannot be adequately interpreted in terms of this restricted conformer population.

The results of normal coordinate calculations on the rotational isomers of 2,4-dichloropentane and 2,4,6-trichloroheptane are reported here. The complete general valence force field used in these calculations predicts well the dominant vibrational frequencies of 2-chloropropane, 2-chlorobutane, 3-chloropentane, and 2,4-dichloropentane (8), as well as the spectrum of PVC and its deuterated derivatives (10). As we will see in the present paper, similar good agreement is obtained for 2,4,6-trichloroheptane. The apparent general success of this potential field, in conjunction with its inability to explain numerous less dominant spectral features in terms of preferred conformers, strongly suggests that other rotational isomers are present.

\section{2,4-DICHLOROPENTANE}

Successful interpretation of the infrared spectra of secondary chlorides depends to a large extent on our ability to analyze the region of the $\mathrm{C}-\mathrm{Cl}$ stretching vibrations. The frequency of this normal mode is sensitive to the geometric arrangement of the substituents attached to the carbon atoms which are joined to the carbon-chlorine bond. It is often possible to predict the presence of certain rotational isomers by comparing the observed $\mathrm{C}-\mathrm{Cl}$ stretching frequencies with the empirically determined value of this frequency as a function of the environment of the carbon-chlorine bond (3). This approach was used to analyze the spectrum of 2,4-dichloropentane.

\section{dl-2,4-Dichloropentane}

In the spectrum of $d l$-2,4-dichloropentane there are two strong absorptions at 606 and $627 \mathrm{~cm}^{-1}$ which are the two ${ }_{\mathrm{H}} S_{\mathrm{H}} \mathrm{C}-\mathrm{Cl}$ stretching vibrations in the preferred ${ }_{\mathrm{H}} S_{\mathrm{H}} S_{\mathrm{H}}$ (TT) conformation (see Table I). In addition, there is a weak band at $715 \mathrm{~cm}^{-1}$ which grows progressively weaker on cooling and is not present in the spectra of crystalline 
Table I. Calculated Wave Numbers (In $\mathrm{cm}^{-1}$ ) and Potential Energy Distributions of Conformers of $d, t-2,4-D 1 c h l o r o p e n t a n e$

\begin{tabular}{|c|c|c|}
\hline $\begin{array}{l}\text { Observed } \\
\text { Wave Number }\end{array}$ & $\begin{array}{l}\text { CaIculated } \\
\text { Wave Number }\end{array}$ & $\begin{array}{l}\text { Potential Energy } \mathrm{b} \\
\text { Distribution }\end{array}$ \\
\hline \multicolumn{3}{|c|}{ 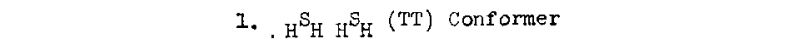 } \\
\hline & $42 \mathrm{~A}^{\mathrm{C}}$ & ${ }^{\top}{ }_{\mathrm{A} 2}(96)$ \\
\hline & $58 \mathrm{~B}$ & $\tau_{\mathrm{H} 2}(90)$ \\
\hline 120 & $120 \mathrm{~A}$ & $\operatorname{ccc}(52), w_{A I}(29)$ \\
\hline \multirow[t]{2}{*}{150} & & $\mathrm{H}_{\mathrm{H} \mathrm{C}_{\mathrm{H}}}^{\mathrm{S}}$ \\
\hline & $240 \mathrm{~A}$ & $\tau_{\mathrm{Al}}(96)$ \\
\hline $245 \mathrm{vw}$ & $241 B$ & $\top^{\top}{ }(91)$ \\
\hline 273 & $267 \mathrm{~A}$ & $W_{A I}(43), x_{\pi A}(39)$ \\
\hline 310 & $312 \mathrm{~B}$ & $x_{\pi B}(44), W_{B I}(33)$ \\
\hline $343 m$ & $342 B$ & $x_{\pi B}(42), w_{B I}^{T}(39)$ \\
\hline $368 \mathrm{ml}$ & $372 \mathrm{~A}$ & $w_{A 2}(65), w_{A 1}(10)$ \\
\hline $460 \mathrm{~m}$ & $458 \mathrm{~B}$ & $w_{\mathrm{B} 2}(74), w_{\mathrm{BI}}(12)$ \\
\hline $476 \mathrm{vw}$ & $472 \mathrm{~A}$ & $x_{\pi A}(28), \operatorname{ccc}(26)$ \\
\hline $606 s$ & $603 \mathrm{~B}$ & $\mathrm{x}_{\mathrm{B}}(84)$ \\
\hline $627 \mathrm{~s}$ & $625 \mathrm{~A}$ & $\mathrm{x}_{\mathrm{A}}(81)$ \\
\hline $650 w$ & & $\mathrm{H}_{\mathrm{H}}^{S_{\mathrm{H}}}, \mathrm{S}_{\mathrm{H}}^{\mathrm{d}}$ \\
\hline $682 w$ & & $\mathrm{H}_{\mathrm{H} \quad \mathrm{C}_{\mathrm{H}}} \stackrel{d}{d}$ \\
\hline $706 w$ & & $\mathrm{H}_{\mathrm{C}}^{\mathrm{S}} \mathrm{H} \mathrm{S}_{\mathrm{H}} \mathrm{d}$ \\
\hline $715 \mathrm{w}$ & & $\mathrm{H}_{\mathrm{C}} \mathrm{C}^{S_{\mathrm{H}}}{ }^{\mathrm{d}}$ \\
\hline \multirow[t]{2}{*}{$877 \mathrm{~ms}$} & $872 \mathrm{~B}$ & $\mathrm{r}(59), \mathrm{B}_{\mathrm{EI}}(23)$ \\
\hline & $884 \mathrm{~A}$ & $\mathrm{R}_{\mathrm{A} \supset}(40), \mathrm{B}_{A 7}(29)$ \\
\hline 938 s & $939 \mathrm{~B}$ & $\mathrm{R}_{\mathrm{BI}}(25), \mathrm{R}_{\mathrm{B} 2}(23)$ \\
\hline $978 \mathrm{~m}$ & $985 \mathrm{~A}$ & $\mathrm{E}_{\mathrm{A} 2}(35), \mathrm{B}_{\mathrm{A} 1}(33)$ \\
\hline $1012 \mathrm{~s}$ & $1023 \mathrm{H}$ & $\mathrm{E}_{\mathrm{B} 2}(61), R_{\mathrm{B} 1}(10)$ \\
\hline $2067 \mathrm{~m}$ & $2068 \mathrm{H}$ & $\mathrm{F}_{\mathrm{BI}}(44), \mathrm{B}_{\mathrm{EI}}(30)$ \\
\hline $1100 \mathrm{vw}$ & $1102 \mathrm{~A}$ & $R_{A 1}(54), t(10)$ \\
\hline $1125 \mathrm{~s}$ & $1119 \mathrm{~A}$ & $\mathrm{R}_{\mathrm{A} 2}(21), \mathrm{B}_{\mathrm{A} 2}(19)$ \\
\hline $1132 \mathrm{~s}$ & $1139 \mathrm{~B}$ & $R_{B 2}(42), H_{\pi B}(10)$ \\
\hline $1191 \mathrm{~m}$ & $1188 \mathrm{~A}$ & $t(28), H_{\sigma A}(23)$ \\
\hline $1222 \mathrm{vvw}$ & $2 \times 606=1212$ & \\
\hline \multirow[t]{2}{*}{$1257 \mathrm{~s}$} & $1252 \mathrm{~B}$ & $\mathrm{H}_{\sigma \mathrm{E}}\langle 85\rangle$ \\
\hline & $1280 \mathrm{IJ}$ & $w(44), \mathrm{E}_{\pi B}(36)$ \\
\hline \multicolumn{3}{|l|}{$1288 \mathrm{~s}$} \\
\hline & $1292 \mathrm{~A}$ & $\mathrm{H}_{\sigma \mathrm{A}}(51), \mathbf{t}(2 \mathrm{~L} j)$ \\
\hline \multirow[t]{2}{*}{$1325 \mathrm{mw}$} & $1325 \mathrm{~A}$ & $\mathrm{H}_{\pi \mathrm{A}}(56), \mathrm{l}_{\mathrm{AI}}(15)$ \\
\hline & $1361 \mathrm{~B}$ & $\mathrm{v}_{\mathrm{b}}(48), w(23)$ \\
\hline $1379 \mathrm{~s}$ & $1381 \mathrm{~A}$ & $\mathrm{U}_{\mathrm{A}}(89)$ \\
\hline
\end{tabular}

samples. This absorption at $715 \mathrm{~cm}^{-1}$ is evidence of the presence of the ${ }_{\mathrm{H}} S_{\mathrm{CC}} S_{\mathrm{H}}$ or $G G$ conformation. The two ${ }_{\mathrm{H}} S_{\mathrm{C}} \mathrm{C}-\mathrm{Cl}$ stretching frequencies of this conformer are calculated at 627 and $714 \mathrm{~cm}^{-1}$. The lower frequency mode is not observed since it is undoubtedly overlapped by the strong absorption at $627 \mathrm{~cm}^{-1}$ of the ${ }_{\mathrm{H}} S_{\mathrm{HH}} S_{\mathrm{H}}$ form. 
Table $[--$ ('ontluned)

\begin{tabular}{ccc}
\hline $\begin{array}{c}\text { Observed } \\
\text { Wave Number }\end{array}$ & $\begin{array}{c}\text { Calculated } \\
\text { Wave Number }\end{array}$ & $\begin{array}{c}\text { Potentlal Energ } \\
\text { O1stribution }\end{array}$ \\
$1415 \mathrm{~ms}$ & $1397 \mathrm{E}$ & $\mathrm{U}_{\mathrm{B}}(46), \mathrm{H}_{\pi \mathrm{E}}(19)$ \\
& $1427 \mathrm{~A}$ & $\delta(95)$ \\
& $1459 \mathrm{~A}$ & $\mathrm{~A}_{\mathrm{Ai}}(72), \mathrm{A}_{\mathrm{A} 2}(18)$ \\
$1443 \mathrm{~s}$ & $1459 \mathrm{~B}$ & $\mathrm{~A}_{\mathrm{BI}}(78), \mathrm{A}_{\mathrm{B} 2}(20)$ \\
$1450 \mathrm{~m}$ & $1459 \mathrm{~B}$ & $\mathrm{~A}_{\mathrm{B} 2}(80), \mathrm{A}_{\mathrm{Bl}}(10)$ \\
& $1460 \mathrm{~A}$ & $\mathrm{~A}_{\mathrm{A} 2}(72), \mathrm{A}_{\mathrm{Al}}(18)$
\end{tabular}

\begin{tabular}{cccc}
\hline $\begin{array}{c}\text { Calculated } \\
\text { Wave Number }\end{array}$ & $\begin{array}{c}\text { Potential Energy } \\
\text { Distribution }\end{array}$ & $\begin{array}{c}\text { Calculated } \\
\text { Wave Number }\end{array}$ & $\begin{array}{c}\text { Potential knergy } \\
\text { Distribution }\end{array}$ \\
\hline
\end{tabular}

2. $\mathrm{H}^{\mathrm{S}} \mathrm{HH} \mathrm{S}_{\mathrm{H}}\left\langle\mathrm{T} \mathrm{G}^{\prime}\right)$ Conformer

\begin{tabular}{|c|c|c|c|}
\hline 41 & $\tau_{A 2}(95)$ & 1012 & ${ }^{2}{ }_{B 1}(32), B_{B 2}(18)$ \\
\hline 45 & ${ }^{\top} \mathrm{A2}(83)$ & 1042 & ${ }_{A 2}(32), R_{A I}(26)$ \\
\hline 136 & $\left(\because C C(53), W_{B 2}(16), W_{B 1}(11)\right.$ & 7090 & $R_{A 1}(32), R_{A 2}(14)$ \\
\hline 228 & $X_{\pi B}(44), W_{B I}(17), \tau_{A I}(14)$ & 1119 & $\mathrm{R}_{\mathrm{B} 1}(33), \mathrm{F}_{\mathrm{AI}}(1 \mathrm{l})$ \\
\hline $24 I$ & $\tau_{B I}(97)$ & 1149 & $\Xi_{\mathrm{B} 2}(17), R_{\mathrm{B} 2}(16)$ \\
\hline 245 & $\tau_{\mathrm{AI}}(80)$ & 1191 & $\mathrm{t}(42), \mathrm{H}_{\mathrm{T}}(31)$ \\
\hline 311 & $W_{A I}(58), X_{\pi A}(23)$ & 1249 & $\operatorname{li}_{\pi A}(8.0)$ \\
\hline 338 & $\mathrm{~W}_{\mathrm{BI}}(46), \mathrm{X}_{\mathrm{TB}}(34)$ & 1276 & $H_{\pi A}(22), H_{\sigma R}(19)$ \\
\hline $37 ?$ & $x_{\pi A}(44), w_{A 1}(20), w_{A 2}(16)$ & 1302 & $\mathrm{H}_{\mathrm{NH}}(27), \mathrm{W}(2 \mathrm{2})$ \\
\hline 418 & $w_{A 2}(50), w_{B 2}(21)$ & 1327 & $I_{\pi Y}\left(f_{3}\right), R_{\mathrm{P}: 1}(18)$ \\
\hline 553 & $\mathrm{x}_{\mathrm{A}}(32), \cos (25), \mathrm{w}_{\mathrm{R} 1}(11)$ & 1362 & ${ }_{A}(31), w(30)$ \\
\hline 615 & $x_{B}(50), x_{A}(48)$ & 1383 & $\tau_{F}(67), v_{A}(21)$ \\
\hline 642 & $x_{B}(46), x_{A}(22)$ & 1389 & $v_{A}(43), B_{B}(16)$ \\
\hline 862 & $R_{A 2}(36), R_{A I}(15)$ & 1432 & $\delta(92)$ \\
\hline 889 & $r(44), R_{A 2}(15)$ & 1459 & $A_{N_{2}}(1), \Lambda_{A 2}(13)$ \\
\hline 918 & $\mathrm{R}_{\mathrm{P} 1}(1 \varepsilon), \mathrm{R}_{\mathrm{B} 2}(I E)$ & 1450 & $A_{11}(97), A_{\mathrm{B} 2}(27)$ \\
\hline \multirow[t]{2}{*}{998} & $I_{A I}(42), B_{2}(17)$ & 1460 & $A_{A:}(70), A_{A I}(14)$ \\
\hline & & 1440 & $A_{\mathrm{R} 2}(57), A_{\mathrm{B} I}(26)$ \\
\hline
\end{tabular}

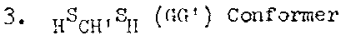

\begin{tabular}{|c|c|c|}
\hline$\tau_{\mathrm{B} 2}(72),{ }_{\mathrm{A} 2}(22)$ & 1011 & $\mathrm{~T}_{\mathrm{HE}}(31), \mathrm{E}_{\mathrm{AI}}(16)$ \\
\hline$\tau_{\mathrm{A} 2}(66), \tau_{\mathrm{B} 2}(24)$ & 1041 & $\mathrm{~B}_{\mathrm{A} 2}(25), \mathrm{R}_{\mathrm{HI}}(22)$ \\
\hline $\operatorname{CCC}(54), \mathrm{w}_{\mathrm{B} 2}(18)$ & 1084 & $\mathrm{R}_{A 2}(16), \mathrm{P}_{\mathrm{A} 2}(14)$ \\
\hline$\tau_{A I}(65), X_{\pi A}(19)$ & 1127 & $R_{A I}(33), r(10)$ \\
\hline
\end{tabular}

At $682 \mathrm{~cm}^{-1}$ an infrared band is observed which is also present in the spectrum of the crystalline phase of $d l$-2,4-dichloropentane. From the results of normal coordinate calculations (Table I and Fig. 1), this band can only be explained by the presence of either the ${ }_{\mathrm{H}} S_{\mathrm{HC}} S_{\mathrm{H}}(T G)$ or the ${ }_{\mathrm{H}} S_{\mathrm{H}^{\prime} \mathrm{H}} S_{\mathrm{H}^{\prime}}\left(G^{\prime} G^{\prime}\right)$ conformations. The Raman spectrum of $d l$-2,4-dichloropentane provides specific evidence that the $682 \mathrm{~cm}^{-1}$ band should be 
Table I--(continued)

\begin{tabular}{|c|c|c|c|}
\hline $\begin{array}{l}\text { Calculated } \\
\text { Wave Number }\end{array}$ & $\begin{array}{l}\text { Potential Energy } \\
\text { Distribution }\end{array}$ & $\begin{array}{l}\text { Calculated } \\
\text { Wave Number }\end{array}$ & $\begin{array}{l}\text { Potential Energy } \\
\text { D1stribution }\end{array}$ \\
\hline 241 & $\tau_{\mathrm{BI}}(97)$ & $115 ?$ & $R_{B C}(28), R_{B I}(14)$ \\
\hline 252 & ${ }^{\tau_{A I}}(29), \mathrm{W}_{\mathrm{BI}}\{29\}$ & 1206 & $t(33), H_{\sigma A}\{23)$ \\
\hline 311 & $\mathrm{w}_{\mathrm{BI}}(50), \mathrm{x}_{\pi \mathrm{A}}(24)$ & 1246 & $\mathrm{H}_{\sigma B}(60), \mathrm{H}_{\sigma A}(28)$ \\
\hline 338 & $x_{\pi B}(72), w_{A I}(14)$ & 1.261 & $H_{\sigma A}(33), H_{\sigma B}(26)$ \\
\hline 350 & $w_{A 1}(39), w_{A 2}(17)$ & 1296 & $\mathrm{H}_{\pi \mathrm{A}}(31), \mathrm{w}(25)$ \\
\hline 420 & $w_{B 2}(41), w_{A 2}(22)$ & 1345 & $\mathrm{H}_{\pi B}(49), t(20)$ \\
\hline 505 & $x_{B}(4 I), w_{A 2}(24)$ & 1363 & $\mathrm{U}_{\mathrm{A}}(42), w(29)$ \\
\hline 624 & $x_{A}(93)$ & 1381 & $\mathrm{U}_{\mathrm{B}}(81)$ \\
\hline 701 & $x_{B}(52), \operatorname{Gcc}(19)$ & 1393 & $U_{A}(43), w(16)$ \\
\hline 867 & $\mathrm{R}_{\mathrm{AI}}(32), \mathrm{R}_{\mathrm{A} 2}(26)$ & 2433 & $\delta(87)$ \\
\hline 895 & $\mathrm{~B}_{\mathrm{A} 1}(32), r(27)$ & 1459 & $A_{A I}(72)$ \\
\hline 899 & $\mathrm{R}_{\mathrm{B} 1}(23), \mathrm{R}_{\mathrm{B} 2}(21)$ & 1460 & $A_{\mathrm{BI}}(54), \mathrm{A}_{\mathrm{B} 2}(23)$ \\
\hline \multirow[t]{2}{*}{979} & $\mathrm{~B}_{\mathrm{BI}}(51), \mathrm{H}_{\pi \mathrm{A}}(17)$ & 1460 & $A_{B 2}(39), A_{A 2}(29)$ \\
\hline & & 1460 & $A_{A 2}(52), A_{B 2}(26)$ \\
\hline
\end{tabular}

4. $\mathrm{H}^{\mathrm{S}} \mathrm{H} \mathrm{H}^{1} \mathrm{~S}_{\mathrm{H}}\left(\mathrm{G}^{1} \mathrm{G}^{2}\right\}$ Conformer

\begin{tabular}{|c|c|c|c|}
\hline $37 \mathrm{~B}$ & $\tau_{B 2}(96)$ & $1007 \mathrm{~A}$ & $\mathrm{~B}_{\mathrm{AI}}(48), \mathrm{B}_{\mathrm{A} 2}(16)$ \\
\hline $51 \mathrm{~A}$ & $\top_{A 2}(89)$ & $1056 \mathrm{~A}$ & $\mathrm{~B}_{\mathrm{A} 2}(45), \mathrm{B}_{\mathrm{A} I}(15)$ \\
\hline $116 A$ & $\operatorname{Ccc}(55), \mathrm{w}_{\mathrm{A} 2}(38)$ & $1079 \mathrm{~B}$ & $\mathrm{~B}_{\mathrm{B} 2}(20), w(18)$ \\
\hline $240 \mathrm{~A}$ & $\tau_{A 1}(94)$ & I083A & $\mathrm{R}_{A I}(38), \mathrm{B}_{\mathrm{BI}}(18)$ \\
\hline $241 B$ & ${ }^{\top} \mathrm{BI}(97)$ & $1157 \mathrm{~B}$ & $\mathrm{R}_{\mathrm{BI}}(27), \mathrm{R}_{\mathrm{B} 2}(17)$ \\
\hline $259 A$ & $w_{A 1}(67), x_{\pi A}(19)$ & $2206 \mathrm{~A}$ & $t(44), H_{O A}(35)$ \\
\hline $294 \mathrm{~B}$ & $x_{\pi B}(73), w_{B I}(11)$ & $1239 B$ & $\mathrm{H}_{\mathrm{OB}}(71), \mathrm{R}_{\mathrm{B} 2}(22)$ \\
\hline $339 \mathrm{~A}$ & $x_{\pi A}(64), w_{A I}(17)$ & $1279 A$ & $\mathrm{H}_{\mathrm{OA}}(53), \mathrm{t}(27)$ \\
\hline $372 B$ & $W_{B 1}(47), W_{B 2}(30)$ & $1294 \mathrm{~B}$ & $\mathrm{H}_{\pi \mathrm{B}}(36), \mathrm{R}_{\mathrm{B} 2}(29)$ \\
\hline $402 B$ & $W_{B 2}(40), W_{B I}(28)$ & $1341 \mathrm{~A}$ & $\mathrm{H}_{\pi \mathrm{A}}(52), \mathrm{R}_{\mathrm{Al}}(19)$ \\
\hline $572 A$ & $x_{A}(75), w_{A I}(20)$ & $1363 \mathrm{~B}$ & $w(49), H_{\pi B}(21)$ \\
\hline $618 \mathrm{~B}$ & $x_{B}(90)$ & $1382 \mathrm{~B}$ & $\mathrm{U}_{\mathrm{B}}(80)$ \\
\hline $689 A$ & $w_{A 2}(34), X_{A}(33)$ & $1385 \mathrm{~A}$ & $U_{A}(83)$ \\
\hline $849 \mathrm{~A}$ & $R_{A 2}(63), R_{A 1}(18)$ & $1435 \mathrm{~A}$ & $\delta(90)$ \\
\hline $887 \mathrm{~B}$ & $\mathrm{R}_{\mathrm{B} 1}(34), \mathrm{r}(28)$ & $1459 \mathrm{~B}$ & ${ }^{A}{ }_{B I}(64), A_{B 2}(26)$ \\
\hline $920 \mathrm{~B}$ & ${ }^{B_{B 2}}(34), r(27)$ & $1459 \mathrm{~A}$ & $A_{A I}(85)$ \\
\hline $979 B$ & $B_{B I}(38), R_{A I}(19)$ & $1460 \mathrm{~B}$ & $A_{B 2}(65), A_{B 1}(26)$ \\
\hline & & $1450 \mathrm{~A}$ & $A_{A 2}(85)$ \\
\hline
\end{tabular}

assigned to the ${ }_{\mathrm{H}} S_{\mathrm{HC}} S_{\mathrm{H}}$ conformation: this follows from the presence of a relatively intense absorption at $150 \mathrm{~cm}^{-1}$ which is only assignable to this conformer. Results of calculations of the normal modes of all six isomers of $d l$-2,4-dichloropentane are given in Table I. For each isomer of 2,4-dichloropentane a band between 100 and $160 \mathrm{~cm}^{-1}$ is predicted. This absorption is close to $150 \mathrm{~cm}^{-1}$ only for the ${ }_{\mathrm{H}} S_{\mathrm{HC}} S_{\mathrm{H}}$ conformer. The 
Tahle I-ricontinued?

\begin{tabular}{|c|c|c|c|}
\hline $\begin{array}{l}\text { Calculated } \\
\text { Wave Number }\end{array}$ & $\begin{array}{l}\text { Foteritial Fnergy } \\
\text { listribution }\end{array}$ & $\begin{array}{l}\text { Calculated } \\
\text { Wave jumber }\end{array}$ & $\begin{array}{l}\text { Fotertial inerg. } \\
\text { Iistribution }\end{array}$ \\
\hline \multicolumn{4}{|c|}{ 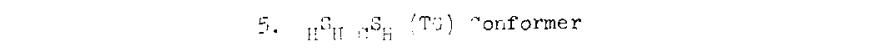 } \\
\hline 47 & $\tau_{B 2}(54), \tau_{A E}(37)$ & 200 & $E_{H I}(3-), E_{A 1}(19)$ \\
\hline 52 & $\tau_{A 2}(48), T_{E 2}(41)$ & 2064 & $P_{\mathrm{H} 2}(2,7), \mathrm{s}_{\mathrm{B} 1}(14)$ \\
\hline 148 & $\operatorname{loc}(47), \mathrm{HI}_{\mathrm{EI}}(30)$ & 10094 & $R_{A 1}(45), R_{n I}(15)$ \\
\hline 228 & $x_{\pi A}(5)$ & 1119 & $R_{E 2}(29), R_{\mathrm{BI}}(2 \varepsilon)$ \\
\hline 241 & $\tau_{A I}(95)$ & 1149 & $R_{A 2}(37), E_{A P}(15)$ \\
\hline 247 & $\top_{B I}(88)$ & 1206 & $t(37), \mathrm{H}_{\mathrm{OE}}(13)$ \\
\hline 275 & $W_{A I}(60)$ & 1241 & $\mathrm{HI}_{\sigma \mathrm{A}}(72), \mathrm{II}_{\sigma \mathrm{E}}(15)$ \\
\hline 343 & $x_{T B}(55), w_{B I}(2 I)$ & 1265 & $\mathrm{H}_{\sigma \mathrm{B}}(55), \mathrm{H}_{\pi \mathrm{B}}(11)$ \\
\hline 395 & $W_{A 2}(26), x_{\pi A}(15)$ & 1301 & $\mathrm{w}(29), \mathrm{H}_{\mathrm{TA}}(19)$ \\
\hline 425 & $w_{A 2}(36), w_{B 2}(17)$ & 1333 & $H_{\pi A}(46), R_{A I}(16)$ \\
\hline 467 & $w_{\mathrm{B} 2}(49), w_{\mathrm{BI}}(15)$ & 1365 & $\mathrm{v}_{B}(56), R_{B 1}(17)$ \\
\hline 614 & $x_{A}(62), x_{B}(31)$ & 1382 & $\mathrm{U}_{\mathrm{A}}(90)$ \\
\hline 675 & $x_{B}(47), x_{A}(35)$ & 1398 & $\mathrm{U}_{\mathrm{B}}(38), \mathrm{H}_{\pi \mathrm{B}}(20)$ \\
\hline 872 & $\mathbf{r}(52), \mathrm{R}_{\mathrm{B} 1}(10)$ & 1.429 & $\delta(91)$ \\
\hline 888 & $\mathrm{R}_{\mathrm{A} 2}(28), \mathrm{B}_{\mathrm{A} 2}(25)$ & 1459 & $A_{A 1}(77), A_{A 2}(13)$ \\
\hline 923 & $\mathrm{~B}_{\mathrm{BE}}(24), \mathrm{R}_{\mathrm{EE}}(22)$ & 1450 & $A_{B 2}(67), A_{B 1}(13)$ \\
\hline \multirow[t]{2}{*}{993} & $B_{A 1}(29), B_{A 2}(26)$ & 1.460 & $A_{B 1}(69), A_{A 2}(13)$ \\
\hline & & 1460 & $A_{A 2}(57), A_{12}(16)$ \\
\hline \multicolumn{4}{|c|}{ 6. $\mathrm{H}^{\mathrm{S}} \mathrm{C} \mathrm{B}_{\mathrm{G}}(\mathrm{GG}$ ) Conformer } \\
\hline $47 E$ & $\tau_{B E}(96)$ & $1002 \mathrm{E}$ & $B_{B I}(29), t(18)$ \\
\hline $57 \mathrm{~A}$ & $T_{A Z}(9 I)$ & $2027 \mathrm{~A}$ & $B_{A 2}(30), B_{B 1}(14)$ \\
\hline $129 A$ & $\operatorname{ccc}(41), W_{A 1}(38)$ & 11258 & $R_{B I}(40), R_{B 2}(18)$ \\
\hline $217 \mathrm{~A}$ & $x_{\pi A}(55), \operatorname{CCC}(12)$ & $1123 \mathrm{~A}$ & $R_{A 2}(37), R_{A 1}(23)$ \\
\hline $241 \mathrm{~A}$ & $\tau_{A I}(97)$ & $2154 \mathrm{~B}$ & $B_{B 2}(20), R_{B 2}(18)$ \\
\hline $242 B$ & $\tau_{B 1}(96)$ & $1217 \mathrm{~A}$ & $t(40), H_{\pi A}(17)$ \\
\hline $31.3 B$ & $\mathrm{~W}_{\mathrm{B} 1}(81)$ & $1242 B$ & $\mathrm{H}_{\mathrm{CB}}(92)$ \\
\hline $345 \mathrm{~A}$ & $x_{\pi A^{(87)}}$ & $1248 \mathrm{~A}$ & $\mathrm{H}_{\sigma \mathrm{A}}(81)$ \\
\hline $371 \mathrm{~A}$ & $\mathrm{x}_{\mathrm{A}}(22), \mathrm{w}_{\mathrm{AI}}(18)$ & $1296 \mathrm{~B}$ & $w(43), U_{\pi B}(37)$ \\
\hline $418 \mathrm{~A}$ & $w_{A 2}(58), w_{A 1}(25)$ & $1348 \mathrm{~A}$ & $\mathrm{H}_{\pi \mathrm{A}}(37), \mathrm{t}(25)$ \\
\hline 4468 & $\mathrm{w}_{\mathrm{B} 2}(69)$ & 23648 & $v_{E}(57), w(17)$ \\
\hline $627 \mathrm{~B}$ & $x_{B}(89), W_{B 1}(10)$ & $1381 \mathrm{~A}$ & $\mathrm{U}_{\mathrm{A}}(8 i)$ \\
\hline $714 \mathrm{~A}$ & $x_{A}(71), \operatorname{ccc}(17)$ & $1399 \mathrm{E}$ & $\mathrm{U}_{\mathrm{B}}(38), w(21)$ \\
\hline $862 B$ & $r(47), R_{B I}(26)$ & $243 \% \mathrm{~A}$ & $8(84)$ \\
\hline $891 \mathrm{~A}$ & $R_{A 2}(28), R_{A \perp}(27)$ & $1459 \mathrm{~A}$ & $A_{A I}(83)$ \\
\hline $906 \mathrm{~B}$ & $\mathrm{~B}_{\mathrm{B} 2}(23), \mathrm{R}_{\mathrm{B} 2}(18)$ & $1460 \mathrm{~B}$ & $A_{B^{2}}(78), A_{B 1}(11)$ \\
\hline \multirow[t]{2}{*}{$99 \mathrm{CA}$} & $E_{A I}(37), E_{D 2}(17)$ & $1460 \mathrm{~B}$ & $A_{B I}(77), A_{B 2}(13)$ \\
\hline & & It4iIA & $n_{A C}(\varepsilon 0)$ \\
\hline
\end{tabular}

See Reference 8 for detalis concerning observed spectra.

${ }^{b}$ See Reference 8 for definitions of coordinates.

$c_{\text {Symmetry species. }}$

dAssigned to small amounts of other conformers. 


\begin{tabular}{|c|c|c|c|c|c|c|}
\hline \multicolumn{7}{|l|}{ dl FORM } \\
\hline CONFORMATION & $T T$ & $\begin{array}{c}T G \\
\text { (GT) }\end{array}$ & $\begin{array}{c}T G^{\prime} \\
\left(G^{\prime} T\right)\end{array}$ & GG & $\begin{array}{r}G G^{\prime} \\
\left(G^{\prime} G\right)\end{array}$ & $G^{*} G^{\prime}$ \\
\hline \multicolumn{7}{|l|}{ STRUCTURE } \\
\hline ISOMERIC STRUCTURES & $S_{H H}, S_{H H}$ & $\mathrm{~S}_{\mathrm{HH}}, \mathrm{S}_{\mathrm{CH}}$ & $S_{H H}, S_{H H^{\prime}}$ & $\mathrm{s}_{\mathrm{CH}}, \mathrm{s}_{\mathrm{CH}}$ & $S_{H^{\prime}}, S_{C H}$ & $\mathrm{~S}_{\mathrm{HH}^{\prime}}, \mathrm{S}_{\mathrm{HH}}$ \\
\hline $\begin{array}{l}\text { CALCULATED } \\
\text { FREOUENCIES }\end{array}$ & $\begin{array}{l}625 \\
603 \\
\end{array}$ & $\begin{array}{l}675 \\
614 \\
\end{array}$ & $\begin{array}{c}642 \\
(615) \\
553\end{array}$ & $\begin{array}{l}714 \\
627 \\
\end{array}$ & $\begin{array}{l}701 \\
624 \\
505\end{array}$ & $\begin{array}{l}689 \\
618 \\
572 \\
\end{array}$ \\
\hline$\frac{\text { MESO FORM }}{\text { CONFORMATION }}$ & TT & $\begin{array}{c}\text { TG } \\
\left(G^{\prime} T\right)\end{array}$ & $\begin{array}{r}T^{\prime} \\
(G T) \\
\end{array}$ & $\begin{array}{c}G G \\
\left(G^{\prime} G^{\prime}\right)\end{array}$ & $G G^{\prime}$ & $G^{\prime} G$ \\
\hline STRUCTURE & & go & & & & \\
\hline ISOMERIC STRUCTURES & $S_{H H}, S_{H H}$ & $S_{H H}, S_{H H^{\prime}}$ & $S_{\mathrm{HH}}, S_{\mathrm{CH}}$ & $\mathrm{S}_{\mathrm{HH}^{\prime}, \mathrm{S}_{\mathrm{CH}}}$ & $s_{\mathrm{CH}}, \mathrm{s}_{\mathrm{CH}}$ & $S_{H^{\prime}}, S_{H H^{\prime}}$ \\
\hline $\begin{array}{l}\text { CALCULATED } \\
\text { FREQUENCIES }\end{array}$ & $\begin{array}{l}(624) \\
604 \\
-\end{array}$ & $\begin{array}{l}649 \\
613 \\
548\end{array}$ & $\begin{array}{l}674 \\
615 \\
-\end{array}$ & $\begin{array}{l}701 \\
620 \\
513\end{array}$ & $\begin{array}{l}714 \\
628 \\
-\end{array}$ & $\begin{array}{c}685 \\
(626) \\
565\end{array}$ \\
\hline
\end{tabular}

FIG. 1. Conformers of $d l$ - and meso-2,4-dichloropentane and their calculated $\mathrm{C}-\mathrm{Cl}$ stretching frequencies ( ) indicates expected weak intensity.

elements of the Jacobian matrix, $\left(\partial \nu_{i} / \partial f_{j}\right)$, which give the sensitivity of each calculated normal frequency $\nu_{i}$, to changes of each force constant $\left(f_{j}\right)$, show that the frequency of the normal vibration between $100 \mathrm{~cm}^{-1}$ and $200 \mathrm{~cm}^{-1}$ in all conformers of 2,4-dichloropentane is relatively invariant with respect to minor force field adjustments. However it is apparent that this frequency is very sensitive to the geometry of the molecule.

Our results also indicate that in addition to the ${ }_{\mathrm{H}} S_{\mathrm{HC}} S_{\mathrm{H}}$ conformer, other minor isomers are present in measurable quantities. Weak bands at $706 \mathrm{~cm}^{-1}$ and $650 \mathrm{~cm}^{-1}$ in the spcctra of crystalline and quenched samples of the $d l$ stereoisomer suggest that the ${ }_{\mathrm{H}} S_{\mathrm{CH}} S_{\mathrm{H}}\left(G G^{\prime}\right)$ and ${ }_{\mathrm{H}} S_{\mathrm{HH}} S_{\mathrm{H}}\left(T G^{\prime}\right)$ forms are also present (see Fig. 1 and Table I).

\section{Meso-2,4-dichloropentane}

In the spectrum of meso-2,4-dichloropentane the strong absorption bands at 611 and $680 \mathrm{~cm}^{-1}$ are assigned to the carbon-chlorine stretching vibrations in the preferred ${ }_{\mathrm{H}} S_{\mathrm{HC}} S_{\mathrm{H}}\left(T G^{\prime}\right)$ conformation (see Table II). However, weak bands at 552 and $645 \mathrm{~cm}^{-1}$ suggest that other rotational isomers are present. From normal coordinate calculations, the $\mathrm{C}-\mathrm{Cl}$ stretching frequencies of the ${ }_{\mathrm{H}} S_{\mathrm{HH}}, S_{\mathrm{H}}(T G)$ isomer of meso-2,4-dichloropentane are predicted at 548,613 , and $649 \mathrm{~cm}^{-1}$ (see Table II). If the predicted frequency at $613 \mathrm{~cm}^{-1}$ (for ${ }_{\mathrm{H}} S_{\mathrm{HH}}, S_{\mathrm{H}}$ ) is overlapped by the strong absorption at $611 \mathrm{~cm}^{-1}$ due to the dominant ${ }_{\mathrm{H}} S_{\mathrm{HC}} S_{\mathrm{H}}$ conformer, the observed bands at $552 \mathrm{~cm}^{-1}$ and $645 \mathrm{~cm}^{-1}$ are 
explained well by admitting the presence of the ${ }_{\mathrm{H}} S_{\mathrm{HH}} S_{\mathrm{H}}$ form of meso-2,4dichloropentane.

In the far infrared and Raman spectra of meso-2,4-dichloropentane there is a relatively strong band at $160 \mathrm{~cm}^{-1}$ which persists at low temperatures. As in the case of the absorption at $150 \mathrm{~cm}^{-1}$ in al-2,4-dichloropentane, we were unable to assign this band to the preferred ${ }_{\mathrm{H}} S_{\mathrm{HC}} S_{\mathrm{H}}$ conformer. The results of normal coordinate calculations on all conformations of meso-2,4-dichloropentane (Table II) suggest that this band is uniquely assignable to a combined skeletal deformation and $\mathrm{C}-\mathrm{C}-\mathrm{Cl}$ bending mode in the ${ }_{\mathrm{H}} S_{\mathrm{H}} S_{\mathrm{H}}$ conformation. A weak infrared absorption at $275 \mathrm{~cm}^{-1}$ in the meso spectrum is also assigned to this rotational isomer. The frequencies of the two ${ }_{\mathrm{H}} S_{\mathrm{HF}} S_{\mathrm{H}} \mathrm{C}-\mathrm{Cl}$ stretching vibrations are calculated at 604 and $625 \mathrm{~cm}^{-1}$. The out-of-phase vibration at $625 \mathrm{~cm}^{-1}$ should be of very low intensity. The $604 \mathrm{~cm}^{-1}$ band is assumed to be overlapped by the strong absorption at $611 \mathrm{~cm}^{-1}$ of the major conformer (viz., $T G^{\prime}$ ).

\begin{tabular}{|c|c|c|}
\hline $\begin{array}{l}\text { Observed } \\
\text { Wave Number }\end{array}$ & $\begin{array}{l}\text { Calculated } \\
\text { Wave Number }\end{array}$ & $\begin{array}{l}\text { Potential Energy } \\
\text { nistribution }\end{array}$ \\
\hline \multicolumn{3}{|c|}{ 1. ${ }_{4} S_{\mathrm{H}} c_{\mathrm{H}}(\mathrm{TC})$ Conformer } \\
\hline & 49 & ${ }^{\top}{ }_{B 2}(82), \tau_{A 2}(12)$ \\
\hline & 56 & $\tau_{A 2}(75), \tau_{\mathrm{P} 2}(13)$ \\
\hline 118 & 129 & 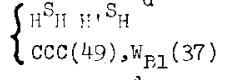 \\
\hline 160 & & $\mathrm{H}^{\mathrm{S}_{H}} \mathrm{II}_{\mathrm{H}}^{\mathrm{S}}{ }^{\mathrm{d}}$ \\
\hline \multirow[t]{2}{*}{228} & 232 & $x_{\pi A}(54), \tau_{B 2}(22)$ \\
\hline & 242 & ${ }^{\top} \mathrm{AL}(96)$ \\
\hline $245 w$ & $2^{1 / 5}$ & $\tau_{B 1}(73)$ \\
\hline $275 w$ & & 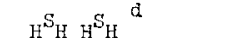 \\
\hline $315 \mathrm{~m}$ & 316 & $w_{A 2}(74)$ \\
\hline $345 \mathrm{w}$ & 342 & $x_{\pi B}(51), w_{B I}(23)$ \\
\hline $392 w$ & 393 & $\mathrm{~W}_{\mathrm{A} 2}(64), x_{\pi \mathrm{B}}(8)$ \\
\hline $410 \mathrm{~ms}$ & 405 & $x_{\pi A}(18), \operatorname{ccc}(15)$ \\
\hline $460 \mathrm{w}$ & 466 & $w_{B 2}(63), w_{B 1}(9)$ \\
\hline 552 & & $\mathrm{H}^{\mathrm{S}} \mathrm{HH}_{\mathrm{H}^{\prime}} \mathrm{S}_{\mathrm{H}}^{\mathrm{d}}$ \\
\hline 611.vs & 615 & $x_{A}(61), x_{B}(42)$ \\
\hline 645 & & ${ }_{H} s_{H I}, S_{H}^{d}$ \\
\hline $680 \mathrm{vs}$ & $6 \% 4$ & $x_{E}(45), x_{A}(37)$ \\
\hline 855 s & 861 & $r(1+2), R_{A 1}(10)$ \\
\hline $88 \% \mathrm{~m}$ & 895 & $\mathrm{I}_{\mathrm{A}_{2}}(25), \mathrm{l}_{\mathrm{Al}}(2 \mathrm{~L})$ \\
\hline $9200^{\circ}$ & 921 & $L_{\mathrm{E}_{1}}(26), \mathrm{K}_{\mathrm{E} 2}(23)$ \\
\hline $980 \mathrm{~ms}$ & 989 & $\mathrm{I}_{1: 1}(31), \mathrm{E}_{\mathrm{A}}(20)$ \\
\hline $1006 \mathrm{~s}$ & 1009 & $\mathrm{~B}_{\mathrm{B2}}(40), \mathrm{B}_{\mathrm{A} 2}(16)$ \\
\hline $1058 s$ & 1059 & $R_{A I}(32), t(13)$ \\
\hline $1089 \mathrm{mw}$ & ingh & $\mathrm{R}_{\mathrm{HI}}(27), \mathrm{R}_{\mathrm{AI}}(15)$ \\
\hline \multirow[t]{2}{*}{$1130 \mathrm{vs}$} & 1132 & $\mathrm{R}_{\mathrm{R} 1}(18), \mathrm{R}_{\mathrm{A} ?}(16)$ \\
\hline & 11.45 & ${ }^{\mathrm{H}}(2), \mathrm{H}_{\mathrm{H} 2}(12)$ \\
\hline
\end{tabular}


Table II--(contlnued)

\begin{tabular}{cll}
$\begin{array}{c}\text { Observed } \\
\text { Wave Number }\end{array}$ & $\begin{array}{c}\text { Calculated } \\
\text { Wave Number }\end{array}$ & $\begin{array}{c}\text { Potent1al vinergy } \\
\text { D1str1but1on }\end{array}$ \\
\hline $1199 \mathrm{~m}$ & 1196 & $t(31), \mathrm{H}_{\sigma \mathrm{A}}(26)$ \\
$123 / \mathrm{s}$ & 1246 & $\mathrm{H}_{\sigma \mathrm{B}}(50), \mathrm{H}_{\sigma \mathrm{A}}(35)$ \\
$1272 \mathrm{~s}$ & 1271 & $\mathrm{H}_{\sigma \mathrm{B}}(30), \mathrm{H}_{\sigma \mathrm{A}}(28)$ \\
$1289 \mathrm{~m}$ & 1291 & $\mathrm{w}(38), \mathrm{H}_{\sigma \mathrm{A}}(28)$ \\
$1337 \mathrm{mw}$ & 1335 & $\mathrm{H}_{\pi \mathrm{A}}(40), \mathrm{t}(22)$ \\
1360 & 1365 & $\mathrm{U}_{\mathrm{B}}(54), \mathrm{R}_{\mathrm{B}}(17)$ \\
$1378 \mathrm{vs}$ & 1382 & $\mathrm{U}_{\mathrm{A}}(88)$ \\
$1415 \mathrm{vw}$ & 1398 & $\mathrm{U}_{\mathrm{B}}(39), \mathrm{H}_{\pi \mathrm{B}}(22)$ \\
$1427 \mathrm{w}$ & 1429 & $\delta(91)$ \\
& 1459 & $\mathrm{~A}_{\mathrm{Al}}(73), \mathrm{A}_{\mathrm{B} 2}(16)$ \\
$1439 \mathrm{~s}$ & 1460 & $\mathrm{~A}_{\mathrm{BI}}(69), \mathrm{A}_{\mathrm{B} 2}(16)$ \\
$1453 \mathrm{~s}$ & 1460 & $\mathrm{~A}_{\mathrm{A} 2}(59), \mathrm{A}_{\mathrm{B} 2}(15)$ \\
& 1460 & $\mathrm{~A}_{\mathrm{B} 2}(42), \mathrm{A}_{\mathrm{A} 2}(29)$ \\
& &
\end{tabular}

\begin{tabular}{cccc} 
Calculated & $\begin{array}{c}\text { Fotential Energy } \\
\text { Distribution }\end{array}$ & $\begin{array}{c}\text { Calculated } \\
\text { Wave Number }\end{array}$ & $\begin{array}{c}\text { Potential Energy } \\
\text { Distribution }\end{array}$ \\
\hline
\end{tabular}

2. ${ }_{\mathrm{H}} \mathrm{S}_{\mathrm{H}} \mathrm{H}_{\mathrm{H}} \mathrm{S}_{\mathrm{H}}(\mathrm{TT})$ Conformer

\begin{tabular}{|c|c|c|c|}
\hline $43 A^{11} \mathrm{C}$ & $\tau_{B 2}(96)$ & $1026 A^{\prime}$ & $\mathrm{B}_{\mathrm{AI}}(32), \mathrm{B}_{\mathrm{AI}}(18)$ \\
\hline $45 A^{\prime}$ & $\tau_{A 2}(82)$ & $2042 \mathrm{~A}^{11}$ & $\mathrm{R}_{\mathrm{BI}}(41), \mathrm{B}_{\mathrm{BC}}(18)$ \\
\hline $153 \mathrm{~A}^{\prime}$ & $\operatorname{coc}(52), w_{A I}(28)$ & $1104 \mathrm{~A}^{\mathrm{t}}$ & $\mathrm{R}_{\mathrm{B} 2}(22), \mathrm{R}_{\mathrm{B} \perp}(20)$ \\
\hline $223 A^{\prime \prime}$ & $x_{\pi B}(53), w_{B}(28)$ & IIIOA' & $\mathrm{R}_{\mathrm{AI}}(57)$ \\
\hline $241 A^{\prime}$ & $\tau_{B}(96)$ & $1160 \mathrm{~A}^{\prime}$ & $\mathrm{R}_{\mathrm{A} 2}(33), \mathrm{B}_{\mathrm{A} 2}(26)$ \\
\hline $247 \mathrm{~A}^{\prime}$ & $\tau_{A}(92)$ & $1.185 A^{\prime \prime}$ & $t(48), \mathrm{H}_{\sigma \mathrm{B}}(28)$ \\
\hline $278 \mathrm{~A}^{\prime}$ & $w_{A}(41), x_{\pi A}(39)$ & $1248 \mathrm{~A}^{\prime}$ & $\mathrm{H}_{\sigma \mathrm{A}}(9 \mathrm{I})$ \\
\hline $342 \mathrm{~A}^{\prime \prime}$ & $\mathrm{x}_{\pi \mathrm{B}}(42), \mathrm{w}_{\mathrm{B} I}(39)$ & $I ว 72 A^{11}$ & $\mathrm{H}_{\pi \mathrm{B}}(35), w(29)$ \\
\hline $409 A^{\prime}$ & $w_{A 2}(66)$ & $1303 A^{\prime \prime}$ & $\mathrm{H}_{\sigma B}(39), t(29)$ \\
\hline $450 A^{\prime \prime}$ & $w_{B 2}(71), w_{B 1}(21)$ & $1328 A^{\prime}$ & $\mathrm{H}_{\pi \mathrm{A}}(70), \mathrm{R}_{\mathrm{Al}}(16)$ \\
\hline $483 A^{\prime}$ & $x_{\pi A}(35), \operatorname{ccc}(24)$ & 1359A" & $\mathrm{U}_{B}(41), w(27)$ \\
\hline $604 A^{\prime}$ & $x_{A}(89)$ & $1381 \mathrm{~A}^{\prime}$ & $\mathrm{v}_{\mathrm{A}}(90)$ \\
\hline $624 A^{\prime \prime}$ & $x_{B}(88)$ & $1395 A^{\prime \prime}$ & $\mathrm{U}_{\mathrm{B}}(52), \mathrm{R}_{\mathrm{B} 2}(15)$ \\
\hline $872 \mathrm{~A}^{\prime}$ & ${ }_{A}{ }_{A I}(40), r(37)$ & $1427 \mathrm{~A}^{\prime}$ & $\delta_{A}(96)$ \\
\hline $89 \subset \mathrm{A}:$ & $\mathrm{R}_{\mathrm{A} 2}(35), \mathrm{B}_{\mathrm{A} 2}(32)$ & $1459 \mathrm{~A}^{\prime}$ & $A_{A 1}(72), A_{B 2}(18)$ \\
\hline $935 \mathrm{~A}^{n}$ & $R_{B 1}(35), R_{B 1}(23)$ & $1460 \mathrm{~A}^{\prime \prime}$ & $A_{B I}(44), A_{A C}(44)$ \\
\hline \multirow[t]{2}{*}{$997 A^{\prime \prime}$} & $\mathrm{B}_{\mathrm{B} 2}(45), \mathrm{B}_{\mathrm{B} 1}(19)$ & $1460 \mathrm{~A}^{\prime}$ & $A_{B 2}(72), A_{A 1}(19)$ \\
\hline & & $1460 \mathrm{~A}^{\prime \prime}$ & $A_{A 2}(45), A_{B I}(45)$ \\
\hline
\end{tabular}

\section{2,4,6-TRICHLOROHEPTANE}

Even with the limitations imposed by the staggered molecular model, each isomer of 2,4,6-trichloroheptane can assume many geometrically unique forms. Thus, in this case a successful interpretation of the infrared spectra relies heavily on our ability to identify the stable structures. 
Table II-(contirucd)

\begin{tabular}{|c|c|c|c|}
\hline $\begin{array}{l}\text { Calculated } \\
\text { Wove Number }\end{array}$ & $\begin{array}{l}\text { Potential Energy } \\
\text { D1stribution }\end{array}$ & $\begin{array}{l}\text { Calculated } \\
\text { Wave Number }\end{array}$ & $\begin{array}{c}\text { Fotential Fnergir } \\
\text { Distribution }\end{array}$ \\
\hline & 3. $\mathrm{F}_{\mathrm{H}} \mathrm{S}_{\mathrm{H}}, \mathrm{S}_{\mathrm{HI}}(\mathrm{T}: \mathrm{T})$ & Conformer & 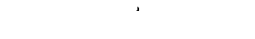 \\
\hline 40 & $T_{\mathrm{B} 2}(96)$ & 1016 & $B_{\mathrm{B} 2}(3 i), \mathrm{B}_{\mathrm{B} I}(17)$ \\
\hline 53 & $\tau_{A 2}(89)$ & 1071 & $\mathrm{~F}_{\mathrm{A} 2}(19), \mathrm{F}_{\mathrm{Al}}(13)$ \\
\hline 1.23 & $\operatorname{ccc}(53), x_{\pi A}(15)$ & 1087 & $\mathrm{R}_{\mathrm{BI}}(31), \mathrm{B}_{\mathrm{B} I}(19)$ \\
\hline 240 & $\tau_{A 1}(89)$ & 1093 & $R_{A 1}(32), R_{A 2}(17)$ \\
\hline 241 & ${ }^{\tau_{B I}}(87)$ & 1153 & $R_{\mathrm{B} 2}(35), R_{\mathrm{B} 1}(14)$ \\
\hline 266 & $\mathrm{w}_{\mathrm{Al}}(51), \mathrm{x}_{\pi \mathrm{B}}(22)$ & 1199 & $t(41), H_{\sigma A}(32)$ \\
\hline 310 & $x_{\pi A}(44), w_{B 1}(36)$ & 1250 & ${ }^{4}(87)$ \\
\hline 336 & $x_{\pi B}(63), w_{A I}(17)$ & 1279 & $w(34), H_{\pi A}(26)$ \\
\hline 356 & $\mathrm{w}_{\mathrm{BI}}(32), \mathrm{x}_{\pi \mathrm{A}}(23)$ & 1287 & ${ }_{\sigma A}^{1}(50), t(29)$ \\
\hline 420 & $w_{A 2}(49), w_{B 2}(19)$ & 1323 & $\mathrm{II}_{\pi B}(49), \mathrm{R}_{\mathrm{E} 1}(14)$ \\
\hline 548 & $x_{A}(33), x_{B}(18)$ & 1362 & $\mathrm{U}_{\mathrm{A}}(30), w(29)$ \\
\hline 613 & $x_{B}(72), x_{A}(28)$ & 1382 & $U_{B}(70), U_{A}(20)$ \\
\hline 649 & $x_{A}(50), w_{A 2}(19)$ & $139 \dot{2}$ & $U_{A}(42), w(15)$ \\
\hline 866 & $\mathrm{R}_{\mathrm{A} 2}(48), \mathrm{R}_{\mathrm{Al}}(13)$ & 1431 & $8(92)$ \\
\hline 889 & $r(49), \mathrm{B}_{\mathrm{Bl}}(15)$ & 1459 & ${ }^{A_{A 1}}(71), A_{B 2}(12)$ \\
\hline 920 & $\mathrm{R}_{\mathrm{B} 2}(23), \mathrm{B}_{\mathrm{Bl}}(18)$ & 1460 & $A_{B I}(46), A_{A 1}(39)$ \\
\hline \multirow[t]{2}{*}{983} & $\mathrm{~B}_{\mathrm{AI}}(32), \mathrm{B}_{\mathrm{A}}(27)$ & 1460 & ${ }_{A}{ }_{A 2}(52), A_{B 1}(36)$ \\
\hline & & 1460 & $A_{B 2}(77), A_{A 1}(13)$ \\
\hline
\end{tabular}

4. $H^{S}+\mathcal{S}_{\mathrm{E}}\left(\hat{G}^{+} \dot{r}^{+}\right)$informer

\begin{tabular}{|c|c|c|c|}
\hline 45 & $\tau_{B 2}(52), \tau_{A 2}(40)$ & 1000 & $\mathrm{~B}_{\mathrm{B} 2}(40), \mathrm{B}_{\mathrm{B} I}(14)$ \\
\hline 50 & $\tau_{A 2}(46), \tau_{B 2}(44)$ & 1048 & $B_{A 2}(26), B_{A I}(13)$ \\
\hline 139 & $\operatorname{CCC}(55), \mathrm{w}_{\mathrm{B} 2}(20)$ & 1080 & $\mathrm{R}_{A 1}(23), \mathrm{B}_{\mathrm{A} 2}(17)$ \\
\hline 231 & $x_{\pi A}(44), w_{A .1}(14)$ & 1130 & $R_{B I}(33), r(13)$ \\
\hline 241 & $T_{A 1}(96)$ & 1146 & $\mathrm{R}_{\mathrm{B} 2}(24), \mathrm{B}_{\mathrm{B} 2}(11)$ \\
\hline 244 & $\tau_{\mathrm{B} 1}(82)$ & 1212 & $\mathrm{t}(39), \mathrm{H}_{\sigma \mathrm{B}}(14)$ \\
\hline 304 & $x_{\pi \mathrm{B}}(36), \mathrm{w}_{\mathrm{A} 1}(33)$ & 1240 & $\mathrm{H}_{\sigma A}\left(7^{\prime} \mathcal{L}\right), \mathrm{H}_{\sigma \mathrm{B}}(1 \mathrm{I})$ \\
\hline 338 & $x_{\pi B}(4 I), w_{B I}(29)$ & 1261 & $\mathrm{H}_{\sigma \mathrm{B}}(59)$ \\
\hline 360 & $\left.x_{\pi A}(4]\right), w_{B I}(29)$ & 132.4 & $w(19), R_{\mathrm{B} 2}(17)$ \\
\hline 419 & $\mathrm{w}_{\mathrm{B} 2}(41), \mathrm{w}_{\mathrm{A} 2}(18)$ & 1327 & $\mathrm{H}_{\pi \mathrm{B}}(50), \mathrm{w}(16)$ \\
\hline 513 & $x_{B}(37), w_{A 2}(31)$ & 1366 & $U_{A}(43), w(1.7)$ \\
\hline 620 & $\mathrm{x}_{\mathrm{A}}(93), \mathrm{w}_{\mathrm{Al}}(12)$ & 1382 & $U_{B}(74), I_{A}(16)$ \\
\hline 701 & $x_{B}(52), \operatorname{ccc}(18)$ & 1393 & $U_{A}(36), H_{\pi D}(18)$ \\
\hline 870 & $R_{A}(28), r(19)$ & 1434 & $\delta(85)$ \\
\hline 879 & $\mathrm{r}(21), \mathrm{R}_{\mathrm{A} 2}(15)$ & 1459 & $A_{A I}(T 2), A_{B I}(10)$ \\
\hline
\end{tabular}

Until recently, as in the case of 2,4-dichloropentane, rotalional isomers of 2,4,6trichloroheptane in which $\mathrm{CH}_{3}$ groups, $\mathrm{CH}_{2}$ and/or chlorine atoms approached one another closely were considered to be sterically forbidden. The presence of different conformations of the stereoisomers of 2,4,6-trichloroheptane can be determined in part by analyzing the $\mathrm{C}-\mathrm{Cl}$ stretching vibrations of the infrared and Raman spectra, and 
Table II--(continued)

\begin{tabular}{cccc}
\hline $\begin{array}{c}\text { Calculated } \\
\text { Wave Number }\end{array}$ & $\begin{array}{c}\text { Potential Energy } \\
\text { Distr1but1on }\end{array}$ & $\begin{array}{c}\text { Calculated } \\
\text { Wave Number }\end{array}$ & $\begin{array}{c}\text { Potential Energy } \\
\text { Distribution }\end{array}$ \\
\hline 911 & $\mathrm{~B}_{\mathrm{BI}}(31), \mathrm{R}_{\mathrm{B} 2}(18)$ & 1460 & $\mathrm{~A}_{\mathrm{B} 1}(47), \mathrm{A}_{\mathrm{A} 2}(29)$ \\
986 & $\mathrm{~B}_{\mathrm{AI}}(32), \mathrm{B}_{\mathrm{A} 2}(21)$ & 1460 & $\mathrm{~A}_{\mathrm{AC}}(37), \mathrm{A}_{\mathrm{B} 1}(29)$ \\
& & 1460 & $\mathrm{~A}_{\mathrm{B} 2}(57), \mathrm{A}_{\mathrm{A} 2}(23)$ \\
\hline
\end{tabular}

5. $\mathrm{H}^{\mathrm{S}} \mathrm{HI}, \mathrm{H}, \mathrm{S}_{\mathrm{HI}}\left(\mathrm{G}^{1} \mathrm{G}\right)$ Conformer

\begin{tabular}{|c|c|c|c|}
\hline $38 A^{\prime \prime}$ & $\tau_{B 2}(97)$ & $1007 \mathrm{~A}^{\prime}$ & $B_{A I}(49), H_{\pi A}(13)$ \\
\hline $46 A^{\prime}$ & $\tau_{A 2}(85)$ & $1029 A^{\prime \prime}$ & $R_{B I}(33), E_{B 1}(29)$ \\
\hline$I 2 I A^{\prime}$ & $\operatorname{ccc}(55), w_{A 2}(37)$ & $1068 \mathrm{~A}^{\prime}$ & $E_{A c^{3}}(51), R_{A 2}(11)$ \\
\hline $231 A^{\prime \prime}$ & $X_{\pi B}(4 I), \tau_{B I}(30)$ & $1133 A^{\prime}$ & $R_{A 1}(39), B_{A 1}(16)$ \\
\hline $241 A^{1}$ & $\tau_{\mathrm{AI}}(96)$ & $1139 A^{\prime \prime}$ & $w(20), H_{\pi B}(14)$ \\
\hline $246 A^{\prime \prime}$ & $\tau_{B I}(65), \mathrm{w}_{\mathrm{BI}}(18)$ & $1199 A^{\prime \prime}$ & $t(40), H_{O B}(34)$ \\
\hline $305 A^{\prime \prime}$ & $w_{B I}(56), x_{\pi B}(34)$ & $1249 A^{\circ}$ & $\mathrm{H}_{\sigma \mathrm{A}}(89)$ \\
\hline $339 A^{\prime}$ & $x_{\pi A}(59), w_{A 1}(22)$ & $1262 \mathrm{~A}^{\prime \prime}$ & $\mathrm{R}_{\mathrm{B} 2}(31), t(22)$ \\
\hline $389 A^{\prime}$ & $\mathrm{w}_{\mathrm{AI}}(59), \mathrm{x}_{\pi \mathrm{A}}(22)$ & $1312 A^{\prime \prime}$ & $\mathrm{H}_{\sigma \mathrm{B}}(28), \mathrm{R}_{\mathrm{B} 2}(17)$ \\
\hline $396 \mathrm{~A}^{\prime}$ & $w_{B 2}(71)$ & $1329 \mathrm{~A}^{\prime}$ & $H_{\pi A}(61), R_{A 2}(18)$ \\
\hline $565 A^{\prime}$ & $\mathrm{x}_{\mathrm{A}}(78), \mathrm{w}_{\mathrm{AI}}(11)$ & $2367 \mathrm{Al}^{\prime \prime}$ & $w(43), H_{\pi \mathrm{B}}(27)$ \\
\hline $626 \mathrm{~A}^{\prime \prime}$ & $x_{B}(89)$ & $1382 A^{\prime \prime}$ & $\mathrm{U}_{\mathrm{H}}(82)$ \\
\hline $685 A^{\prime}$ & $W_{A 2}(35), x_{A}(28)$ & $1385 \mathrm{~A}^{\prime}$ & $U_{A}(84)$ \\
\hline $851 A^{\prime}$ & $\mathrm{R}_{\mathrm{AC}}(59), \mathrm{R}_{\mathrm{Al}}(21)$ & $1435 \mathrm{~A}^{\prime}$ & $8(92)$ \\
\hline 897A" & $R_{\mathrm{BI}}(29), \mathrm{B}_{\mathrm{BI}}(27)$ & $1459 A^{\prime}$ & $A_{B I}(71), A_{A C}(18)$ \\
\hline 90eA' & $r(50), B_{A 2}(21)$ & $1459 A^{\prime \prime}$ & $A_{A 1}(80)$ \\
\hline \multirow[t]{2}{*}{$997 \mathrm{~A}^{\prime \prime}$} & ${ }^{\mathrm{B}} \mathrm{P} 2(55), \mathrm{B}_{\mathrm{B} 1}(19)$ & $1460 \mathrm{~A}^{\prime \prime}$ & $A_{B 2}(81)$ \\
\hline & & $1460 \mathrm{~A}$ & $A_{A P}(72), A_{B 1}(19)$ \\
\hline
\end{tabular}

6. ${ }_{\mathrm{H}} \mathrm{S}_{\mathrm{C}} \mathrm{C}_{\mathrm{HT}}\left(\mathrm{GC} \mathrm{F}^{\prime}\right)$ Conformer

\begin{tabular}{|c|c|c|c|}
\hline $55 A^{\prime}$ & $\tau_{A 2}(88)$ & $1017 \mathrm{~A}^{\prime \prime}$ & $\mathrm{B}_{\mathrm{B} 2}(42), \mathrm{R}_{\mathrm{BI}}(27)$ \\
\hline $56 A^{\prime \prime}$ & $T_{F 2}(96)$ & $1021 \mathrm{~A}^{\prime}$ & $\mathrm{B}_{\mathrm{A} 2}(52) \cdot \mathrm{B}_{\mathrm{AI}}(14)$ \\
\hline $137 \mathrm{~A}^{\prime}$ & $\cos (36), \mathrm{W}_{\mathrm{Al}}(29)$ & $1116 \mathrm{~A}^{\prime \prime}$ & $\mathrm{R}_{\mathrm{B} 2}(31), \mathrm{R}_{\mathrm{B} 1}(23)$ \\
\hline $225 A^{\prime}$ & $x_{\pi A}(46), \operatorname{coc}(15)$ & $2123 \mathrm{~A}^{\prime}$ & $R_{A 1}(40), R_{A 2}(22)$ \\
\hline $24 I A^{\prime \prime}$ & $\tau_{\mathrm{BI}}(98)$ & $1149 \mathrm{~A}^{\prime}$ & $R_{A 2}(20), B_{A 1}(16)$ \\
\hline $248 \mathrm{~A}^{\circ}$ & ${ }^{\top}{ }_{A 1}(84)$ & $1213 \mathrm{~A}^{\prime \prime}$ & $t(34), w(14)$ \\
\hline $274 A^{\prime \prime}$ & $\mathrm{w}_{\mathrm{B} 1}(89)$ & $1245 A^{\prime \prime}$ & $\mathrm{H}_{\mathrm{OB}}(85)$ \\
\hline $346 A^{\prime \prime}$ & $x_{\pi B}(88)$ & $1247 \mathrm{~A}^{\prime}$ & $\mathrm{H}_{\mathrm{OA}}(90)$ \\
\hline $370 A^{\prime}$ & $W_{A 2}(22), x_{A}(21)$ & $1321 \mathrm{~A}^{\prime}$ & $H_{\pi A}(63), R_{A 1}(17)$ \\
\hline $438 A^{\prime}$ & $W_{A 2}(44), W_{A I}(31)$ & $1322 A^{* 1}$ & $w(47), t(23)$ \\
\hline
\end{tabular}

by means of NMR. In addition, the probable geometry assumed by adjacent carbonchlorine bonds in 2,4,6-trichloroheptane can be obtained from knowledge of this relative orientation in 2,4-dichloropentane. We have calculated the normal vibration frequencies of the ${ }_{\mathrm{H}} S_{\mathrm{HH}} S_{\mathrm{HH}} S_{\mathrm{H}}(T T T T)$ and ${ }_{\mathrm{H}} S_{\mathrm{HH}} S_{\mathrm{CC}} S_{\mathrm{H}}(T T G G)$ conformers of syndiotactic 2,4,6-trichloroheptane. For the isotactic isomer the ${ }_{\mathrm{H}} S_{\mathrm{HC}} S_{\mathrm{HC}} S_{\mathrm{H}}(T G T G),{ }_{\mathrm{H}} S_{\mathrm{CH}} S_{\mathrm{HC}} S_{\mathrm{H}}$ 
Table $11--($ concluded)

\begin{tabular}{|c|c|c|c|}
\hline $\begin{array}{l}\text { Calculated } \\
\text { Wave Number }\end{array}$ & $\begin{array}{l}\text { Totent1al Energy } \\
\text { Distribution }\end{array}$ & $\begin{array}{l}\text { Calculated } \\
\text { Wave Number }\end{array}$ & $\begin{array}{l}\text { Potent1al Ener } \\
\text { D1stribution }\end{array}$ \\
\hline $440 A^{11}$ & $\mathrm{w}_{\mathrm{B} 2}(78)$ & $1369 \mathrm{~A}^{\prime \prime}$ & $\mathrm{U}_{\mathrm{B}}(67), \mathrm{R}_{\mathrm{B} I}(17)$ \\
\hline $628 A^{\prime \prime}$ & $x_{B}(89)$ & $1382 \mathrm{~A}^{\circ}$ & $U_{A}(89)$ \\
\hline $714 A^{\prime}$ & $x_{A}(71), \operatorname{CCC}(17)$ & $1400 \mathrm{~A}^{11}$ & $\mathrm{U}_{\mathrm{B}}(28), \mathrm{H}_{\pi \mathrm{P}}(24)$ \\
\hline $866 A^{\prime}$ & $r(45), R_{A I}(29)$ & $1431 \mathrm{~A}^{\prime}$ & $8(87)$ \\
\hline $898 A^{\prime \prime}$ & $\mathrm{R}_{\mathrm{Bl}}(26), \mathrm{R}_{\mathrm{B} 2}(25)$ & $1459 \mathrm{~A}^{\circ}$ & $A_{A 1}(81)$ \\
\hline $898 A^{2}$ & $\mathrm{~B}_{\mathrm{AI}}(43), \mathrm{R}_{\mathrm{A} 2}(21)$ & $1460 \mathrm{~A}^{\prime \prime}$ & $\Lambda_{A 2}\left(8^{\prime} l\right)$ \\
\hline \multirow[t]{2}{*}{$989 A^{\prime \prime}$} & $\mathrm{B}_{\mathrm{B} 1}(41), \mathrm{B}_{\mathrm{B} 2}(21)$ & $1460 \mathrm{~A}^{\prime \prime}$ & $A_{B I}(83)$ \\
\hline & & $1461 \mathrm{~A}^{\prime}$ & $A_{\mathrm{B} 2}(79)$ \\
\hline
\end{tabular}

\footnotetext{
$a_{\text {See Reference }} 8$ for detalls concerning observed spectra.

$b_{\text {Bee Reference }} 8$ for definitions of coordinates.

$c_{\text {Symmetry species. }}$

dissigned to small amounts of other conformers.
}

$\left(G^{\prime} T T G\right),{ }_{\mathrm{H}} S_{\mathrm{HH}} S_{\mathrm{HH}} S_{\mathrm{H}}\left(T T T G^{\prime}\right)$, and the ${ }_{\mathrm{H}} S_{\mathrm{HH}} S_{\mathrm{HH}} S_{\mathrm{H}}(T T T T)$ conformers were all considered. The spectrum of heterotactic 2,4,6-trichloroheptane is well accounted for by two conformations, ${ }_{\mathrm{H}} S_{\mathrm{HH}} S_{\mathrm{HC}} S_{\mathrm{H}}(T T T G)$ and ${ }_{\mathrm{H}} S_{\mathrm{HH}} S_{\mathrm{CH}} S_{\mathrm{H}}\left(T T G^{\prime} T\right)$. The observed and calculated frequencies for 2,4,6-trichloroheptane are given in Tables III, IV, and V. The infrared spectra for 2,4,6-trichloroheptane were reported by Shimanouchi, Tasumi, and Abe (6) and by Doskočilova, et al. (11). The observed spectra used here are a combination of these observations.

The $\mathrm{C}-\mathrm{Cl}$ stretching region of the spectrum reported by Doskočilova et al., for the syndiotactic form of 2,4,6-trichloroheptane contains a strong band at $608 \mathrm{~cm}^{-1}$ which decreases in intensity at low temperature and develops a shoulder at $604 \mathrm{~cm}^{-1}$, and a strong band at $642 \mathrm{~cm}^{-1}$ which shifts to $632 \mathrm{~cm}^{-1}$ and increases in intensity as the sample solidifies. No bands were reported in the region of $\mathrm{CS}_{\mathrm{H}}$ type $\mathrm{C}-\mathrm{Cl}$ stretching vibrations. For syndiotactic 2,4,6-trichloroheptane, $\mathrm{C}-\mathrm{Cl}$ stretching frequencies were calculated at $604 \mathrm{~cm}^{-1}, 616 \mathrm{~cm}^{-1}$, and $629 \mathrm{~cm}^{-1}$ for the ${ }_{\mathrm{H}} S_{\mathrm{HH}} S_{\mathrm{HH}} S_{\mathrm{H}}(T T T T)$ conformer and at $610 \mathrm{~cm}^{-1}, 631 \mathrm{~cm}^{-1}$, and $722 \mathrm{~cm}^{-1}$ for the ${ }_{\mathrm{H}} S_{\mathrm{H}} S_{\mathrm{CC}} S_{\mathrm{H}}(T T G G)$ form. The observed bands are assigned as follows: the band at $604 \mathrm{~cm}^{-1}$ is assigned to the predicted band at $604 \mathrm{~cm}^{-1}$; the band at $608 \mathrm{~cm}^{-1}$ is assigned to the overlapped $\mathrm{C}-\mathrm{Cl}$ stretching vibrations calculated at $616 \mathrm{~cm}^{-1}$ for the all trans ${ }_{\mathrm{H}} S_{\mathrm{HH}} S_{\mathrm{HH}} S_{\mathrm{H}}$ conformer and at $610 \mathrm{~cm}^{-1}$ for the ${ }_{\mathrm{H}} S_{\mathrm{HH}} S_{\mathrm{CC}} S_{\mathrm{H}}$ conformer; the $632 \mathrm{~cm}^{-1}$ band is assigned to the predicted $629 \mathrm{~cm}^{-1}$ band in ${ }_{\mathrm{H}} S_{\mathrm{HH}} S_{\mathrm{HH}} S_{\mathrm{H}}$ conformer and to the $631 \mathrm{~cm}^{-1}$ band of the ${ }_{\mathrm{H}} S_{\mathrm{HH}} S_{\mathrm{CC}} S_{\mathrm{H}}$ conformation. Although not reported as such, some weak absorption in the region $725 \mathrm{~cm}^{-1}$ can be observed in the spectrum of syndiotactic 2,4,6-trichloroheptane recorded by Doskoxilova et al. This is assignable to the ${ }_{\mathrm{C}} S_{\mathrm{H}} \mathrm{C}-\mathrm{Cl}$ stretching vibration of the ${ }_{\mathrm{H}} S_{\mathrm{HH}} S_{\mathrm{CC}} S_{\mathrm{H}}$ isomer, which is calculated at $722 \mathrm{~cm}^{-1}$.

The region of the $\mathrm{C}-\mathrm{Cl}$ stretching vibrations in the spectrum of isotactic 2,4,6trichloroheptane contains strong bands at $619 \mathrm{~cm}^{-1}$ and $688 \mathrm{~cm}^{-1}$. At elevated temperatures a shoulder at $635 \mathrm{~cm}^{-1}$ is present which disappears at low temperatures. There 


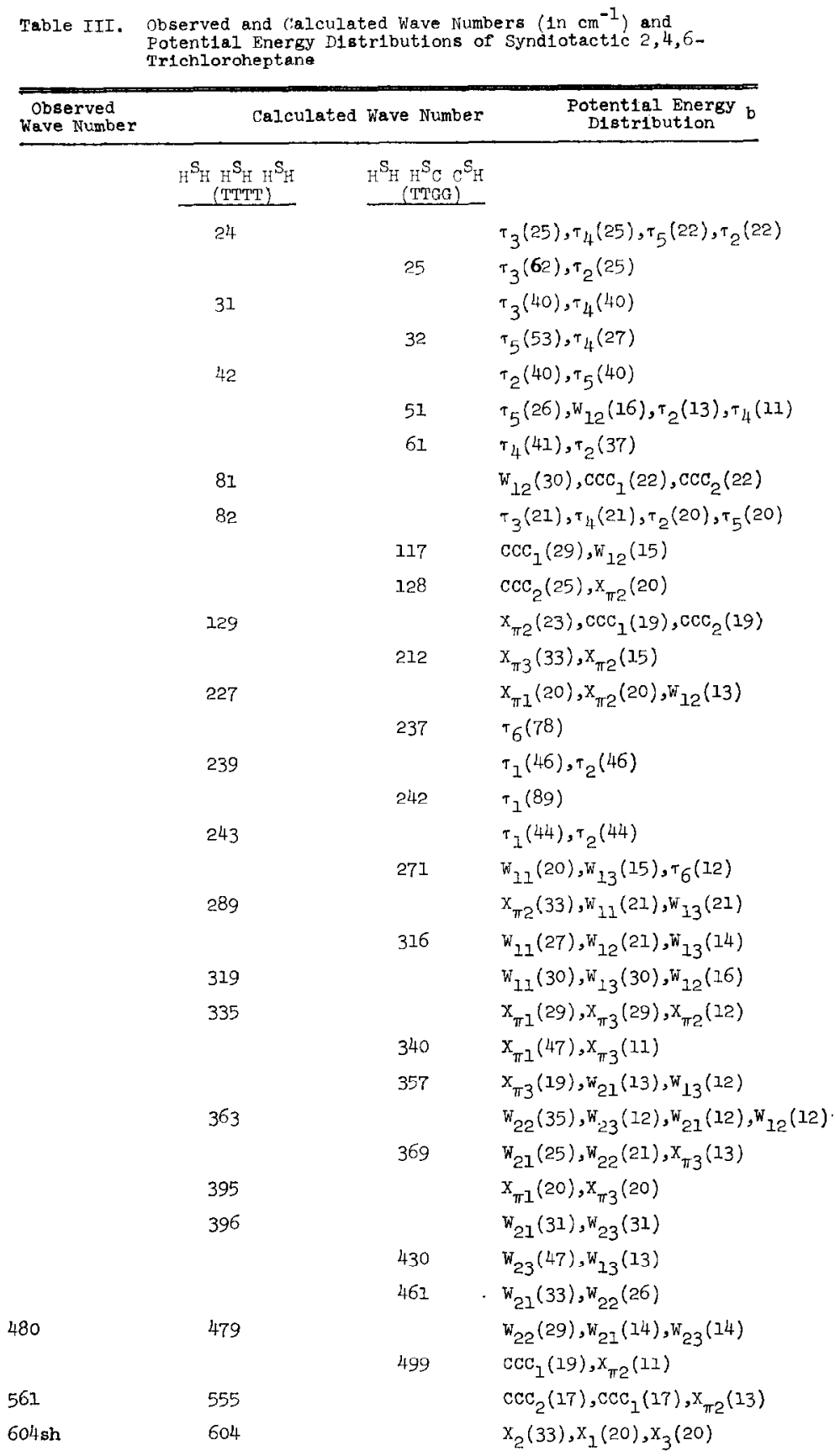

is also a band at approximately $563 \mathrm{~cm}^{-1}$ which has a similar temperature dependence. The predicted ${ }_{\mathrm{H}} S_{\mathrm{H}} \mathrm{C}-\mathrm{Cl}$ stretching vibrations for the ${ }_{\mathrm{H}} S_{\mathrm{HC}} S_{\mathrm{HC}} S_{\mathrm{H}}$ and ${ }_{\mathrm{H}} S_{\mathrm{CH}} S_{\mathrm{HC}} S_{\mathrm{H}}$ isomers are at $614 \mathrm{~cm}^{-1}$ and $619 \mathrm{~cm}^{-1}$, respectively. These are jointly assigned to the observed band at $619 \mathrm{~cm}^{-1}$. The four $\mathrm{c}_{\mathrm{H}} \mathrm{C}-\mathrm{Cl}$ stretching vibrations are predicted at 
T'able III--(continued

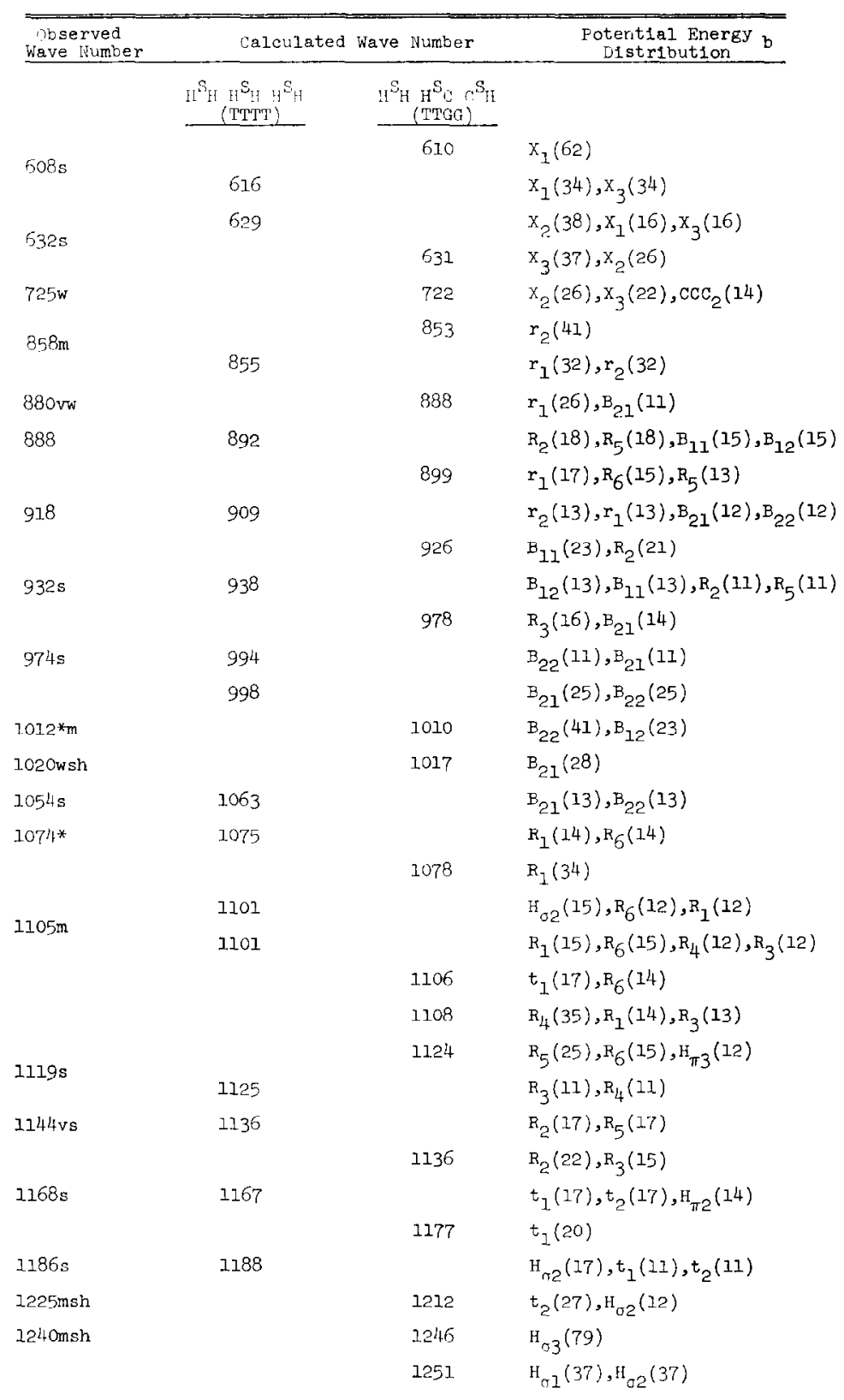


Tabie III--(concluded $)$

\begin{tabular}{|c|c|c|c|}
\hline $\begin{array}{c}\text { Observed } \\
\text { Wave Number }\end{array}$ & Calculated & Wave Number & $\begin{array}{l}\text { Potential Energy } \\
\text { D1stribution }\end{array}$ \\
\hline & 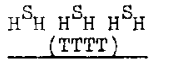 & 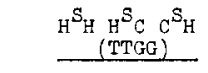 & \\
\hline $1253 \mathrm{~s}$ & 1256 & & $\mathrm{H}_{u 1}(28), \mathrm{H}_{\sigma 3}(28), \mathrm{H}_{\sigma 2}(24)$ \\
\hline $1265 \mathrm{vs}$ & 1257 & & $\mathrm{H}_{\pi 2}(25), w_{1}(22), w_{2}(22)$ \\
\hline \multirow[t]{3}{*}{$1274 \mathrm{~m}$} & 1273 & & $H_{\sigma 1}(29), H_{\sigma 3}(29)$ \\
\hline & & 1282 & $w_{1}(28), H_{\pi 2}(13), w_{2}(11)$ \\
\hline & & 1283 & $\mathrm{H}_{\sigma 1}(21), \mathrm{t}_{1}(14), \mathrm{H}_{\pi 1}(14), \mathrm{H}_{\pi 2}(14$ \\
\hline $1298 * \mathrm{vw}$ & 1293 & & $\mathrm{H}_{\pi 3}(17), \mathrm{H}_{\pi 1}(17), w_{2}(13), w_{1}(13)$ \\
\hline 1305 vwsh & & 1309 & $t_{1}(20), w_{2}(20)$ \\
\hline \multirow{4}{*}{$1317 \mathrm{~m}$} & 1312 & & $t_{1}(21), t_{2}(21), H_{02}(21)$ \\
\hline & 1322 & & $\mathrm{H}_{\pi 1}(20), \mathrm{H}_{\pi 3}(20), \mathrm{H}_{\pi 2}(1.5)$ \\
\hline & & 1338 & $t_{2}(17), H_{\pi 1}(15), H_{\pi 3}(12)$ \\
\hline & & 1358 & $\mathrm{U}_{2}(16), \mathrm{w}_{2}(11)$ \\
\hline \multirow[t]{4}{*}{1355 vwsh } & 1362 & & $\mathrm{U}_{1}(15), \mathrm{U}_{2}(15), \mathrm{w}_{1}(14), w_{2}(14)$ \\
\hline & 1369 & & $\mathrm{U}_{1}(2 \mathrm{I}), \mathrm{U}_{2}(21), \mathrm{H}_{\pi 2}(12)$ \\
\hline & & 1373 & $\mathrm{U}_{1}(27), \mathrm{U}_{2}(27)$ \\
\hline & & 1388 & $U_{1}(34), U_{2}(26)$ \\
\hline \multirow[t]{3}{*}{$1380 \mathrm{~s}$} & 1390 & & $\mathrm{v}_{1}(27), \mathrm{v}_{2}(27)$ \\
\hline & 1393 & & $\mathrm{v}_{1}(18), \mathrm{U}_{2}(18)$ \\
\hline & & 1397 & $w_{2}(14), U_{2}(13)$ \\
\hline \multirow[t]{7}{*}{$1422 \mathrm{~m}$} & 1424 & & $\delta_{1}(46), \delta_{2}(46)$ \\
\hline & & 1426 & $\delta_{1}(90)$ \\
\hline & 1429 & & $\delta_{2}(47), \delta_{2}(47)$ \\
\hline & & 1434 & $\delta_{2}(77)$ \\
\hline & 1.459 & & $A_{11}(39), A_{22}(39)$ \\
\hline & 1459 & & $A_{22}(36), A_{11}(36)$ \\
\hline & 1459 & & $A_{12}(43), A_{21}(43)$ \\
\hline \multirow[t]{5}{*}{$1448 s$} & 1459 & & $A_{12}(42), A_{21}(42)$ \\
\hline & & 1459 & $A_{11}(69), A_{21}(18)$ \\
\hline & & 1459 & $A_{12}(73), A_{22}(15)$ \\
\hline & & 1459 & $A_{21}(72), A_{11}(19)$ \\
\hline & & 1460 & $A_{22}(72), A_{12}(16)$ \\
\hline
\end{tabular}

* Also assigned to the neterotactic stereolsomers.

a References 6 and 11 .

b See Reference 8 for definitions of coordinates. 
Table IV. Observed and 'alcilated wave Jumkers ill $\mathrm{cm}^{-1}$, ard Fotential Energy listribitions of Isotactic $4,4-$ irichloroheptane

\begin{tabular}{|c|c|c|c|}
\hline $\begin{array}{l}\text { Observed } \\
\text { Wave Number }\end{array}$ & Calculated & Wave Number & $\begin{array}{c}\text { Potential Trergy b } \\
\text { Jistribition }\end{array}$ \\
\hline & 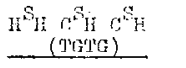 & 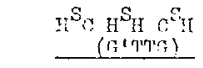 & \\
\hline & 24 & & $\tau_{3}(41), \tau_{2}(38), \tau_{5}(12)$ \\
\hline & & 31 & $\tau_{2}(25), \tau_{5}(25), \dot{\tau}_{3}(23), \tau_{4}(23)$ \\
\hline & 32 & & $\tau_{4}(59) \cdot \tau_{5}(25)$ \\
\hline & & 35 & $\tau_{3}(34), \tau_{4}(34)$ \\
\hline & & 46 & $\tau_{2}(36), \tau_{5}(36)$ \\
\hline & 52 & & $\tau_{2}(30), \tau_{4}(16), W_{12}(16), \tau_{5}(12)$ \\
\hline & 61 & & $\tau_{3}(41), \tau_{5},(34)$ \\
\hline & & 70 & $w_{12}(28), \operatorname{ccc}_{1}(20), \operatorname{ccc}_{2}(20)$ \\
\hline & & 78 & $\tau_{3}(23), \tau_{4}(23), \tau_{2}(16), \tau_{5}(16)$ \\
\hline & 105 & & $\operatorname{ccc}_{1}(22)+H_{12}(18)$ \\
\hline & 137 & & $\mathrm{CC}_{2}(31), \mathrm{x}_{\pi 2}(24), \operatorname{coc}_{1}(13), \mathrm{w}_{13}(11)$ \\
\hline & & 153 & $x_{\pi 2}(21), \cos _{1}(17), \operatorname{coc}_{2}(17)$ \\
\hline & & 189 & $x_{\pi 1}(22), x_{\pi 3}(2)$, \\
\hline & 223 & & $x_{\pi 2}(19), x_{\pi 3}(17), \tau_{6}(12)$ \\
\hline & & 235 & $\tau_{1}(37), \tau_{6}(37)$ \\
\hline & 239 & & $\tau_{6}(45), \tau_{1}(43)$ \\
\hline & & 240 & $\tau_{1}(47), \tau_{6},(47)$ \\
\hline & 247 & & $\tau_{6}(35), \tau_{7}(33)$ \\
\hline & $25 \%$ & & $w_{11}(23), x_{\pi 3}(22)$ \\
\hline & & 266 & $x_{\pi 2}(19), x_{\pi 1}(15), r_{\pi 3}(15)$ \\
\hline & 310 & & $w_{12}(31), W_{13}(24)$ \\
\hline & & 316 & $w_{11}(30), w_{13}(30), w_{12}(20)$ \\
\hline & & 335 & $x_{\pi 3}(24), x_{\pi 1}(24), w_{11}(13), w_{13}(13)$ \\
\hline & 338 & & $w_{11}(26), x_{\pi 3}(24), w_{13}(19)$ \\
\hline & & 361 & $W_{12}(16), W_{22}(16), x_{\pi 1}(11), x_{\pi 2}(11)$ \\
\hline & 376 & & $W_{21}(22), x_{\pi 1}(16)$ \\
\hline & 384 & & $w_{22}(14), x_{\pi 2}(12), w_{13}(11), x_{\pi 1}(11)$ \\
\hline & & 387 & $w_{22}(23), w_{21}(15), w_{23}(15)$ \\
\hline & $4 \perp 1$ & & $w_{21}(18), x_{2}(15), x_{\pi 1}(14), w_{22}(12)$ \\
\hline $425 w$ & & 424 & $w_{21}(30), w_{23}(30)$ \\
\hline \multirow[t]{3}{*}{$450 w$} & 440 & & $w_{23}(34)$ \\
\hline & & 472 & $\begin{array}{l}x_{\pi 2}(23), x_{1}(16), x_{2}(16), \\
\operatorname{ccc}_{1}(14), \operatorname{ccc}_{2}(14)\end{array}$ \\
\hline & & 483 & $w_{22}(27), w_{21}(16), w_{23}(16)$ \\
\hline $495 \mathrm{~m}$ & 197 & & $w_{22}(2 I), w_{21}(14)$ \\
\hline
\end{tabular}


Table IV--(cont1nued)

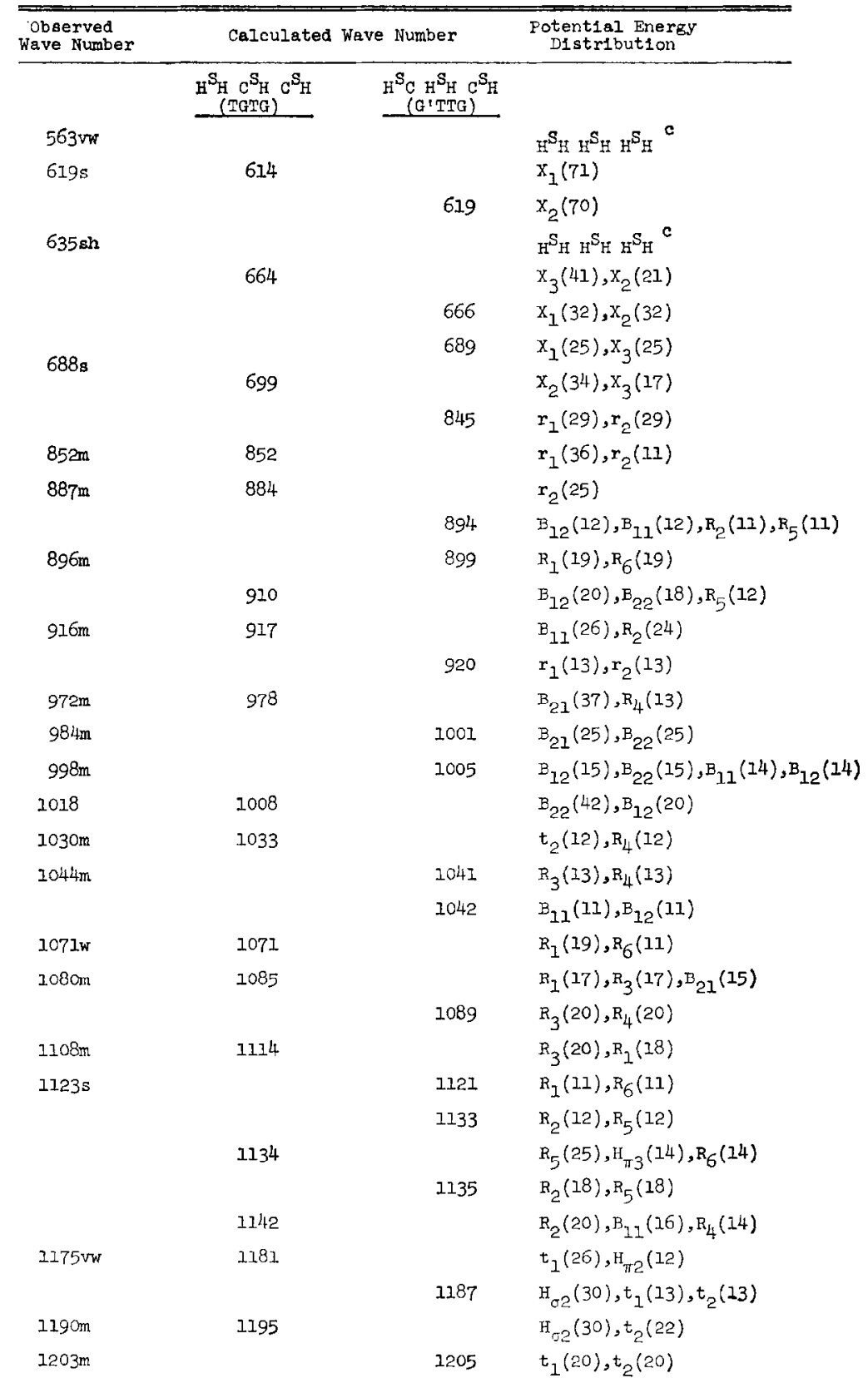


'jable L $v--($ continiced)

\begin{tabular}{|c|c|c|c|}
\hline $\begin{array}{l}\text { Observed } \\
\text { Wave Number }\end{array}$ & Calculated & Wave number & $\begin{array}{l}\text { Potential Fneres } \\
\text { Distribution }\end{array}$ \\
\hline & $\begin{array}{l}\mathrm{H}^{\mathrm{S}_{\mathrm{H}}} \mathrm{C}_{\mathrm{H}} \mathrm{C}^{\mathrm{S}_{\mathrm{H}}} \\
\text { (TITG) }\end{array}$ & $\begin{array}{l}\mathrm{H}^{\mathrm{S}} \mathrm{C}_{\mathrm{Y}} \\
\end{array}$ & \\
\hline $1233 s$ & & $10 / 3$ & $H_{\tau I}(36), H_{\pi 3}(36)$ \\
\hline \multirow[t]{2}{*}{$1243 \mathrm{~s}$} & $22 / 17$ & & $\mathrm{H}_{53}(76)$ \\
\hline & & 1249 & $\mathrm{H}_{\sigma 1}(39), \mathrm{H}_{a 3}(39)$ \\
\hline \multirow[t]{2}{*}{$1252 \mathrm{~s}$} & 2.262 & & $\mathrm{H}_{01}(35), \mathrm{H}_{02}(25)$ \\
\hline & & 1265 & $\mathrm{H}_{\pi 2}(28), \mathrm{w}_{1}(19), \mathrm{w}_{2}(19)$ \\
\hline \multirow[t]{2}{*}{$1277 \mathrm{~m}$} & 1268 & & $\mathrm{H}_{\sigma 2}(21), \mathrm{H}_{\sigma 1}(17), \mathrm{w}_{2}(11)$ \\
\hline & & 1281 & $\mathrm{H}_{02}(42)$ \\
\hline $1288 \mathrm{~m}$ & 1290 & & $\mathrm{H}_{\pi 1}(28), w_{1}(25)$ \\
\hline \multirow[t]{3}{*}{$1312 \mathrm{~m}$} & 1311 & & $w_{2}(26), t_{1}(17), H_{\pi I}(11)$ \\
\hline & & 1328 & $t_{2}(15), t_{1}(15), w_{1}(14), w_{2}(14)$ \\
\hline & & 1732 & $H_{\pi 1}(16), H_{\pi 3}(16)$ \\
\hline $133^{\prime}+\mathrm{w}$ & 1336 & & $1 I_{\pi 3}(17), t_{2}(15), \mathrm{H}_{\pi 2}(11)$ \\
\hline \multirow[t]{4}{*}{$1353 w$} & 1357 & & $w_{1}(14), U_{2}(13), v_{1}(11)$ \\
\hline & & $136+$ & $\mathrm{U}_{2}(2 \mathrm{I}), \mathrm{U}_{1}(2 \mathrm{I})$ \\
\hline & & $13 \%$ & $\mathrm{U}_{1}(27), \mathrm{U}_{2}(27)$ \\
\hline & 1374 & & $U_{P}(3 I), U_{1}(3 I)$ \\
\hline \multirow[t]{3}{*}{$1385 \mathrm{~s}$} & 1388 & & $\mathrm{U}_{1}(32), \mathrm{U}_{2}(32)$ \\
\hline & & 1392 & $\mathrm{u}_{1}(22), \mathrm{v}_{2}(22)$ \\
\hline & & 1325 & $v_{1}(13), v_{2}(13), H_{\pi 2}(11)$ \\
\hline \multirow[t]{3}{*}{$1415 \mathrm{vw}$} & 1399 & & $w_{1}(11), w_{2}(11)$ \\
\hline & & $1+27$ & $\delta_{I}(145), \delta_{2}(45)$ \\
\hline & 14128 & & $\delta_{2}(70), \delta_{2}(18)$ \\
\hline \multirow[t]{2}{*}{$1430 \mathrm{msh}$} & & 2430 & $\delta_{2}(45), x_{2}(45)$ \\
\hline & $1 / 31$ & & $\delta_{1}(65), 5_{2}(19)$ \\
\hline \multirow[t]{8}{*}{$1447 \mathrm{~s}$} & $1 / 459$ & & $A_{11}(59), A_{12}(16), A_{P I}(11)$ \\
\hline & 1459 & & $A_{12}(57), A_{12}(15), A_{22}(12)$ \\
\hline & & 1459 & $A_{11}(36), A_{21}(36)$ \\
\hline & & 1459 & $A_{21}(39), A_{11}(39)$ \\
\hline & 1459 & & $A_{2 I}(75), A_{11}(16)$ \\
\hline & 1460 & & $A_{20}\left(7^{\prime}+\right), A_{12}(16)$ \\
\hline & & 1460 & $A_{22}(73)$ \\
\hline & & 1460 & $A_{12}(69)$ \\
\hline
\end{tabular}


Table IV--(cont Inued)

\begin{tabular}{|c|c|c|c|}
\hline \multirow[t]{2}{*}{$\begin{array}{c}\text { Observed } \\
\text { Wave Number }\end{array}$} & \multicolumn{2}{|c|}{ Calculated Wave Number } & \multirow[t]{2}{*}{$\begin{array}{l}\text { Potent1al Energy } \\
\text { D1stribution }\end{array}$} \\
\hline & 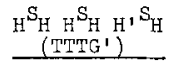 & 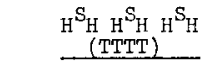 & \\
\hline & 23 & & $\tau_{4}(37), \tau_{2}(28), \tau_{5}(19)$ \\
\hline & & 25 & $T_{3}(24), \tau_{2}(24), \tau_{4}(24), T_{5}(24)$ \\
\hline & & $3 I$ & $\tau_{3}(42), \tau_{2}(42)$ \\
\hline & 31. & & $\mathrm{~T}_{3}(55), \mathrm{T}_{4}(30)$ \\
\hline & & 38 & $\tau_{2}(36), \tau_{5}(36)$ \\
\hline & 43 & & $\tau_{5}(43), \tau_{2}(39)$ \\
\hline & 60 & & $\tau_{5}(17), \tau_{4}(16), \tau_{3}(16), \mathrm{Ccc}_{1}(13)$ \\
\hline & & 63 & $\tau_{3}(19), \tau_{4}(19), \tau_{2}(16), \tau_{5}(16)$ \\
\hline & 88 & & $\operatorname{ccc}_{2}(32), \mathrm{w}_{12}(16)$ \\
\hline & & 92 & $\operatorname{ccc}_{1}(21), \operatorname{ccc}_{2}(21), \mathrm{w}_{12}(19)$ \\
\hline & 262 & & $\operatorname{ccc}_{1}(22), x_{\pi 2}(15), \operatorname{ccc}_{2}(11)$ \\
\hline & & 194 & $w_{12}(32), x_{\pi 3}(22), x_{\pi 1}(22)$ \\
\hline & & 201 & $\operatorname{ccc}_{1}(17), \operatorname{ccc}_{2}(17), \mathrm{w}_{13}(13), \mathrm{w}_{11}(13)$ \\
\hline & & 210 & $x_{\pi 2}(60)$ \\
\hline & 213 & & $x_{\pi 1}(34), w_{12}(28)$ \\
\hline & 233 & & $x_{\pi 2}(37), w_{11}(18)$ \\
\hline & 242 & & ${ }^{\top} G^{(88)}$ \\
\hline & & 243 & $\tau_{1}(48), \tau_{6}(48)$ \\
\hline & 244 & & $\tau_{1}(89)$ \\
\hline & & 246 & $\tau_{1}(4 b), \tau_{6}(45)$ \\
\hline & & 279 & $w_{13}(32), w_{11}(32)$ \\
\hline & 315 & & $x_{\pi 1}(26), w_{11}(23), w_{13}(22)$ \\
\hline & & 325 & $x_{\pi 1}(26), x_{\pi 3}(26), w_{11}(17), w_{13}(17)$ \\
\hline & 331 & & $x_{\pi 3}(65)$ \\
\hline & 358 & & $w_{13}(33), w_{23}(15)$ \\
\hline & & 392 & $w_{23}(16), w_{21}(16)$ \\
\hline & 411 & & $w_{21}(40), w_{22}(23)$ \\
\hline & & 417 & $w_{21}(32), w_{23}(32)$ \\
\hline & & 429 & $w_{22}(34)$ \\
\hline & 444 & & $w_{22}(30), w_{21}(24)$ \\
\hline & 457 & & $x_{\pi 1}(20), \operatorname{coc}_{1}(16), W_{23}(11)$ \\
\hline & & 469 & $W_{22}(21), W_{21}(15), W_{23}(15)$ \\
\hline & 568 & & $x_{3}(43)$ \\
\hline & & 565 & $\operatorname{cCc}_{1}(19), \operatorname{CCC}_{2}(19), \mathrm{x}_{\pi 2}(16)$ \\
\hline & & 604 & $x_{2}(36), x_{1}(20), x_{3}(20)$ \\
\hline
\end{tabular}


Table IV--(contluned)

\begin{tabular}{|c|c|c|c|}
\hline \multirow[t]{2}{*}{$\begin{array}{l}\text { Observed } \\
\text { Wave Number }\end{array}$} & \multicolumn{2}{|c|}{ Calculated Wave Number } & \multirow[t]{2}{*}{$\begin{array}{l}\text { Fotential Energy } \\
\text { Iictribution }\end{array}$} \\
\hline & 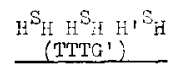 & $\underbrace{\mathrm{H}^{\mathrm{S}} \mathrm{H} \mathrm{H}^{\mathrm{S}} \mathrm{S}_{\mathrm{H}} \mathrm{H}^{\mathrm{S}} \mathrm{H}}$ & \\
\hline & 607 & & $x_{1}(45), x_{2}(31)$ \\
\hline & & 613 & $x_{3}(36), x_{1}(36)$ \\
\hline & 623 & & $x_{2}(1 \mid 0), x_{1}(29)$ \\
\hline & & 628 & $x_{2}(37), x_{2}(16), x_{3}(16)$ \\
\hline & 660 & & $x_{3}(38), w_{23}(19), \operatorname{cCc}_{2}(12)$ \\
\hline & & 862 & $r_{1}(35), r_{2}(27)$ \\
\hline & 865 & & $r_{1}(30), r_{2}(30)$ \\
\hline & 883 & & $R_{5}(25), R_{G}(16)$ \\
\hline & & 893 & $R_{2}(19), R_{5}(19), B_{11}(15), B_{21}(15)$ \\
\hline & & 912 & $\mathrm{~B}_{12}(14), \mathrm{B}_{22}(14), \mathrm{r}_{1}(11), \mathrm{r}_{2}(1 \mathrm{I})$ \\
\hline & 921 & & $B_{I I}(23), R_{2}(16)$ \\
\hline & 932 & & $r_{2}(25), r_{1}(16)$ \\
\hline & & $9 / 41$ & $\mathrm{~B}_{11}(14), \mathrm{B}_{21}(14)$ \\
\hline & 970 & & $\mathrm{H}_{22}(35), \mathrm{R}_{4}(16), \mathrm{H}_{\pi 3}(13)$ \\
\hline & & 1003 & $\mathrm{E}_{12}(26), \mathrm{E}_{22}(26)$ \\
\hline & & 1011 & $R_{3}(16), R_{4}(16), R_{1}(16), R_{6}(16)$ \\
\hline & 1013 & & $B_{12}(40)$ \\
\hline & & 1027 & $\mathrm{~B}_{12}(18), \mathrm{B}_{22}(18)$ \\
\hline & 1031 & & $B_{22}(18), R_{1}(14)$ \\
\hline & 1071 & & $\mathrm{~B}_{12}(27), \mathrm{R}_{6}(12)$ \\
\hline & & 1071 & $R_{G}(17) \cdot R_{1}(17)$ \\
\hline & 1075 & & $\mathrm{R}_{3}(23), \mathrm{R}_{4}(16)$ \\
\hline & & 1092 & $\mathrm{R}_{4}(25), \mathrm{R}_{3}(25)$ \\
\hline & 1100 & & $R_{1}(38)$ \\
\hline & & 1105 & $\mathrm{R}_{I}(20), \mathrm{R}_{6}(20)$ \\
\hline & & 1113 & $R_{1}(12), R_{6}(12), R_{2}(11), R_{5}(11)$ \\
\hline & 1121 & & $R_{2}(19), B_{11}(14), R_{3}(12)$ \\
\hline & 1146 & & $R_{6}(20), R_{14}(18), R_{5}(15)$ \\
\hline & & 2154 & Iess than $10 \%$ \\
\hline & & 1183 & $t_{1}(25), t_{2}(25)$ \\
\hline & 2185 & & $t_{1}(41), R_{2}(14), H_{o I}(1 I)$ \\
\hline & & 1186 & $t_{2}(20), t_{1}(20), \mathrm{H}_{02}(20)$ \\
\hline & 1192 & & $t_{2}(31), H_{02}(16), H_{03}(12)$ \\
\hline & 1243 & & $H_{\sigma 1}(26), H_{\sigma 2}(15)$ \\
\hline & . & 2245 & $H_{\sigma 1}(29), M_{\sigma 3}(19), 4_{\sigma 2}(16)$ \\
\hline
\end{tabular}


Table IV--(concluded)

\begin{tabular}{|c|c|c|c|}
\hline \multirow[t]{2}{*}{$\begin{array}{l}\text { bserved } \\
\text { Wave Ifumber }\end{array}$} & \multicolumn{2}{|c|}{ Calculated Wave Number } & $\begin{array}{l}\text { Potential Energy } \\
\text { Distribution }\end{array}$ \\
\hline & 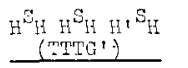 & 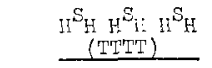 & \\
\hline & & $121+7$ & $\mathrm{H}_{\sigma 1}(20), \mathrm{H}_{\sigma 3}(20), \mathrm{H}_{\sigma 2}(14)$ \\
\hline & 1257 & & $\mathrm{H}_{\sigma 3}(40), \mathrm{H}_{\pi 2}(14), w_{1}(11)$ \\
\hline & 1268 & & $\mathrm{H}_{01}(26), \mathrm{t}_{2}(14), \mathrm{H}_{\sigma 3}(11)$ \\
\hline & & +282 & $\begin{array}{l}H_{\sigma 3}(20), H_{\pi 1}(20), t_{1}(11), \\
t_{2}(11),\end{array}$ \\
\hline & & 2294 & $\begin{array}{l}\mathrm{H}_{\pi 1}(2 I), \mathrm{H}_{\pi 2}(21), \mathrm{w}_{1}(10), \\
\mathrm{w}_{2}(10),\end{array}$ \\
\hline & 1305 & & $\mathrm{H}_{\sigma 2}(18), \mathrm{H}_{\pi I}(17), \mathrm{t}_{1}(14)$ \\
\hline & 1309 & & $\mathrm{H}_{\pi T}(18), \mathrm{w}_{y}(11)$ \\
\hline & & 1316 & $\mathrm{H}_{\sigma 2}(24), \mathrm{t}_{1}(16), \mathrm{t}_{2}(16)$ \\
\hline & & 1327 & $\mathrm{H}_{\pi 1}(23), \mathrm{H}_{\pi 3}(23), \mathrm{H}_{\pi 2}(19)$ \\
\hline & 1336 & & ${ }^{11} \pi_{3}(29), t_{2}(11)$ \\
\hline & & 1360 & $\mathrm{v}_{1}(14), \mathrm{U}_{2}(14), \mathrm{w}_{2}(14), \mathrm{w}_{1}(14)$ \\
\hline & 1360 & & $w_{1}(22), U_{1}(20), w_{2}(12)$ \\
\hline & & 1366 & $\mathrm{H}_{\pi 2}(16), \mathrm{U}_{1}(15), \mathrm{U}_{2}(15)$ \\
\hline & 1370 & & $w_{2}(22), H_{\pi 2}(17), R_{14}(12)$ \\
\hline & 1383 & & $\mathrm{v}_{2}(70)$ \\
\hline & & .1389 & $\mathrm{U}_{2}(28), \mathrm{U}_{1}(28)$ \\
\hline & & 1390 & $U_{1}(24), U_{2}(24)$ \\
\hline & 1390 & & $\mathrm{U}_{1}(50), \mathrm{w}_{1}(11)$ \\
\hline & & 2425 & $\delta_{1}(47), \delta_{2}(47)$ \\
\hline & 1426 & & $. \delta_{1}(81), \delta_{2}(13)$ \\
\hline & & 1428 & $\delta_{1}(47), \delta_{2}(47)$ \\
\hline & 1431 & & $8_{2}(78), \delta_{1}(13)$ \\
\hline & 1459 & & $A_{11}(60), A_{12}(19)$ \\
\hline & & 1459 & $A_{22}(65), A_{21}(18)$ \\
\hline & 1459 & & $A_{21}(66)$ \\
\hline & & 1459 & $A_{11}(34), A_{21}(34)$ \\
\hline & 1459 & & $A_{22}(76), A_{21}(15)$ \\
\hline & & 1459 & $A_{12}(60), A_{11}(23)$ \\
\hline & 2459 & & $A_{12}(69), A_{11}(22)$ \\
\hline & & 1459 & $A_{21}(34), A_{11}(34)$ \\
\hline
\end{tabular}

a References 6 and 11 .

. See Reference 8 for definitions of coordinates.

c Assigned to another conformer. 


\begin{tabular}{|c|c|c|c|}
\hline $\begin{array}{l}\text { Observed } \\
\text { Wave Number }\end{array}$ & Calculated & Wave Irumber & $\begin{array}{l}\text { Potential Fnergy } b \\
\text { Distribution }\end{array}$ \\
\hline & 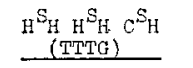 & 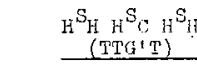 & \\
\hline & & 25 & $\tau_{4}(32), \tau_{2}(31), \tau_{3}(16), \tau_{5}(15)$ \\
\hline & & 25 & $\tau_{3}(47), \tau_{5}(31)$ \\
\hline & 26 & & $\tau_{3}(49), \tau_{5}(21), \tau_{2}(18)$ \\
\hline & 34 & & $\tau_{4}(54), \tau_{2}(15)$ \\
\hline & 45 & & $\tau_{5}(44), T_{2}(34)$ \\
\hline & & 58 & $\tau_{2}(50), \tau_{4}(17)$ \\
\hline & & 59 & $\tau_{5}(34), \tau_{4}(27)$ \\
\hline & 73 & & $\mathrm{~W}_{12}(22), \mathrm{CCc}_{2}(18), \mathrm{CCC}_{1}(16), \tau_{4}(11)$ \\
\hline & 83 & & $\tau_{3}(17), \tau_{2}(15), \tau_{5}(11)$ \\
\hline & & 105 & $\operatorname{cCC}_{1}(21), W_{12}(18), \operatorname{cCc}_{2}(14)$ \\
\hline & & 1.30 & $\operatorname{ccc}_{2}(22), x_{\pi 2}(20), \operatorname{ccc}_{1}(19)$ \\
\hline & 139 & & $x_{\pi 2}(21), \operatorname{ccc}_{1}(19), \operatorname{ccc}_{2}(16)$, \\
\hline & $\cdot$ & & $x_{\pi 1}(12), w_{13}(12)$ \\
\hline & 207 & & $x_{\pi 3}(31)$ \\
\hline & & 233 & $\tau_{1}(24), x_{\pi 2}(22), \tau_{6}(12), x_{\pi 3}(12)$ \\
\hline & 238 & & $\tau_{6}(83)$ \\
\hline & & 239 & $\tau_{6}(65), \tau_{2}(26)$ \\
\hline & 241 & & $\tau_{1}(87)$ \\
\hline & & 246 & $\tau_{1}(46), \tau_{6}(13), x_{\pi 3}(14)$ \\
\hline & & 271 & $x_{\pi I}(20), W_{13}(19), W_{11}(14)$ \\
\hline & 281 & & $\mathrm{w}_{11}(22), \mathrm{x}_{\pi 2}(21), \mathrm{w}_{13}(15)$ \\
\hline & & 314 & $w_{11}(31), w_{12}(22)$ \\
\hline & 316 & & $w_{11}(27), w_{13}(19), W_{12}(18)$ \\
\hline & 335 & & $x_{\pi 3}(29), x_{\pi 1}(24), W=13(14)$ \\
\hline & & 338 & $W_{13}(35), x_{\pi 1}(28)$ \\
\hline & & 362 & $w_{21}\left(3^{\prime}\right), w_{22}(17), w_{11}(13)$ \\
\hline & 366 & & $w_{22}(31), w_{12}(14), x_{\pi 1}(12)$ \\
\hline & 374 & & $W_{21}(31), x_{\pi 3}(11)$ \\
\hline & & 394 & $x_{\pi 3}(31), x_{\pi 1}(14)$ \\
\hline & & 409 & $w_{23}(38)$ \\
\hline & 415 & & $w_{23}(36), w_{21}(18), w_{13}(11)$ \\
\hline $460 \mathrm{~m}$ & & 461 & $w_{21}(32), w_{22}(25)$ \\
\hline \multirow[t]{2}{*}{$480 \mathrm{~m}$} & 480 & & $w_{22}(28), w_{23}(18), w_{21}(11)$ \\
\hline & & 514 & $w_{23}(14), \mathrm{Ccc}_{1}(13)$ \\
\hline
\end{tabular}


Table V--(continued)

\begin{tabular}{|c|c|c|c|}
\hline $\begin{array}{l}\text { Observed } \\
\text { Wave Number }\end{array}$ & Calculated & Wave Number & $\begin{array}{l}\text { Potential Energy } \\
\text { Distribution }\end{array}$ \\
\hline \multirow{3}{*}{$525 \mathrm{~m}$} & 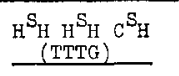 & 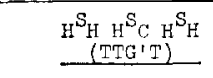 & \\
\hline & 518 & & $\operatorname{ccc}_{1}(20), \mathrm{x}_{3}(18), \mathrm{x}_{\pi 2}(15)$ \\
\hline & 607 & & $x_{2}(44), x_{2}(27)$ \\
\hline $610 \mathrm{~s}$ & & 611 & $x_{1}(53), x_{3}(16)$ \\
\hline 620 & & 616 & $x_{3}(55), x_{1}(17)$ \\
\hline $628 \mathrm{sh}$ & 626 & & $x_{2}(43), x_{1}(27)$ \\
\hline \multirow{2}{*}{$689_{s}$} & 680 & & $x_{3}(54), w_{13}(11)$ \\
\hline & & 689 & $x_{2}(54), W_{12}(11)$ \\
\hline \multicolumn{4}{|l|}{$810 \mathrm{w}$} \\
\hline \multirow[t]{2}{*}{$850 \mathrm{~m}$} & 850 & & $r_{2}(33), r_{1}(28)$ \\
\hline & & 855 & $r_{2}(40)$ \\
\hline \multirow{2}{*}{$881 \mathrm{~m}$} & & 886 & $r_{I}(4 I), B_{2 I}(13)$ \\
\hline & 888 & & $R_{5}(16), R_{6}(12)$ \\
\hline $910 \mathrm{msh}$ & & 910 & $B_{12}(22), R_{5}(16)$ \\
\hline \multirow[t]{3}{*}{$917 \mathrm{~m}$} & 916 & & $\mathrm{~B}_{22}(14), \mathrm{B}_{12}(13), \mathrm{r}_{2}(12)$ \\
\hline & 923 & & $\mathrm{R}_{2}(18), \mathrm{r}_{2}(17), \mathrm{B}_{11}(15)$ \\
\hline & & 931 & $R_{2}(16), B_{11}(16)$ \\
\hline $967 \mathrm{~s}$ & & 975 & $\mathrm{~B}_{22}(28), \mathrm{R}_{3}(13)$ \\
\hline $984 \mathrm{~s}$ & 993 & & $B_{21}(26), B_{22}(13)$ \\
\hline \multirow{3}{*}{$1009 \mathrm{~m}$} & & 1005 & $\mathrm{~B}_{21}(29), \mathrm{B}_{22}(12)$ \\
\hline & 1012 & & $\mathrm{~B}_{22}(29), \mathrm{B}_{12}(\mathrm{I} 8)$ \\
\hline & 1030 & & $R_{4}(23), t_{2}(11)$ \\
\hline $1054 \mathrm{~m}$ & & 1059 & $R_{3}(12)$ \\
\hline $1072 \mathrm{~m}$ & & 1069 & $R_{6}(21), R_{4}(15), R_{1}(15)$ \\
\hline $2083 \mathrm{vw}$ & $108 I$ & & $R_{1}(27), R_{6}(12)$ \\
\hline \multirow{2}{*}{ 1090vw } & 1090 & & $\mathrm{R}_{3}(33)$ \\
\hline & & 1094 & $R_{1}(26), R_{6}(17)$ \\
\hline $1105 \mathrm{w}$ & 2105 & & $R_{4}(20), R_{1}(14), t_{1}(14)$ \\
\hline \multirow{5}{*}{$1122 \mathrm{~s}$} & & 11117 & $R_{4}(20), R_{2}(12), R_{6}(11)$ \\
\hline & & 1120 & $t_{2}(20)$ \\
\hline & $11 \cdot 31$ & & $\mathrm{R}_{3}(16), \mathrm{R}_{2,}(15)$ \\
\hline & 1136 & & $\mathrm{~K}_{5}(2 \mathrm{I}), \mathrm{R}_{2}(13)$ \\
\hline & & 11.40 & $\mathrm{k}_{3}(19), \mathrm{k}_{2}(11)$ \\
\hline $116 \%$ rwsh & & 2164 & $k_{5}(15), t_{2}(14), t_{1}(11)$ \\
\hline $1183 \mathrm{~m}$ & 1179 & & $t_{1}\left(2^{\prime}\right), H_{02}(12)$ \\
\hline $1204 \mathrm{~mm}$ & 1196 & & $t_{2}(31), H_{v 2}(13)$ \\
\hline
\end{tabular}


Table $v-$ - concluded

\begin{tabular}{|c|c|c|c|}
\hline \multirow[t]{2}{*}{$\begin{array}{l}\text { Observed } \\
\text { Wave IIumber }\end{array}$} & \multicolumn{2}{|c|}{ (alculated Wave Number } & \multirow[t]{2}{*}{$\begin{array}{l}\text { Jntential linergy } \\
\text { Jistribution }\end{array}$} \\
\hline & 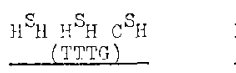 & 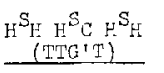 & \\
\hline & & 1197 & $\mathrm{H}_{\sigma 2}(18), \mathrm{t}_{2}\left(1^{\prime} /\right), \mathrm{H}_{\sigma 3}(12), \mathrm{t}_{1}(11$ \\
\hline $1230 \mathrm{msh}$ & $\begin{array}{l}2 \times 628=1256 \\
2 \times 610=1220\end{array}$ & & \\
\hline $1245 \mathrm{~s}$ & 1246 & & $\mathrm{H}_{\sigma 3}(71)$ \\
\hline $3255 \mathrm{msh}$ & & 1251 & $\mathrm{H}_{\sigma 2}(40), \mathrm{H}_{\sigma 1}(35)$ \\
\hline $125 \operatorname{sins}$ & 1261 & & $w_{1}(21), I_{\pi 2}(20), w_{2}(17)$ \\
\hline \multirow{2}{*}{$1273 \mathrm{~s}$} & & 1263 & $\mathrm{H}_{\pi 3}(48), \mathrm{H}_{\pi 2}(11)$ \\
\hline & 1263 & & $\mathrm{H}_{\sigma 1}(51), \mathrm{H}_{\sigma 2}(16)$ \\
\hline \multirow{4}{*}{$1287 \mathrm{~m}$} & & 1282 & $\mathrm{H}_{\sigma I}(19), \mathrm{H}_{\pi I}(13), \mathrm{t}_{1}(11)$ \\
\hline & 1290 & & $\mathrm{H}_{\sigma 2}(23), \mathrm{H}_{\pi 1}(14)$ \\
\hline & & 1290 & $\mathrm{H}_{\pi 3}(19), \mathrm{w}_{1}(15), \mathrm{w}_{2}(13)$ \\
\hline & & 1303 & $t_{1}(21), w_{2}(12)$ \\
\hline $1314 \mathrm{~m}$ & $131_{14}$ & & $t_{1}(24), H_{\pi I}(23)$ \\
\hline $1322 \mathrm{~m}$ & & 1329 & $\mathrm{H}_{\pi 1}(16), \mathrm{H}_{\pi 2}(15), \mathrm{t}_{2}(15)$ \\
\hline $1348 \mathrm{vw}$ & 1332 & & $\mathrm{t}_{2}(22), \mathrm{II}_{\pi}(2 \mathrm{l})$ \\
\hline \multirow[t]{3}{*}{$1358 w$} & & 1356 & $w_{1}(14), w_{2}(13), U_{1}(11)$ \\
\hline & 1363 & & $\mathrm{U}_{1}(19), \mathrm{w}_{1}(16), \mathrm{U}_{2}(15)$ \\
\hline & 1371 & & $\mathrm{U}_{2}(32), \mathrm{U}_{1}(1.7), \mathrm{H}_{\pi 2}(1.1)$ \\
\hline \multirow{5}{*}{13838} & & 1373 & $u_{2}(29), u_{1}\left(2^{\prime}\right)$ \\
\hline & & 1388 & $\mathrm{U}_{1}(35), \mathrm{U}_{2}(28)$ \\
\hline & 1391 & & $v_{1}(37), v_{2}(13), w_{1}(11)$ \\
\hline & 1395 & & $v_{2}(22), w_{2}(14)$ \\
\hline & & 1397 & $v_{2}(14), w_{2}(11)$ \\
\hline \multirow{2}{*}{1423} & 1425 & & $\delta_{3}(67), \delta_{2}(25)$ \\
\hline & & 1426 & $\delta_{1}(83)$ \\
\hline \multirow{2}{*}{1438 wh } & 1430 & & $\delta_{2}(6+), \delta_{1}(26)$ \\
\hline & & 1431 & $\varepsilon_{2}(75)$ \\
\hline \multirow[t]{4}{*}{$1448 \mathrm{~s}$} & 1459 & & $A_{11}(45), A_{21}(25), A_{12}(14)$ \\
\hline & & 1459 & $A_{11}(40), A_{21}(28), A_{12}(13)$ \\
\hline & 1459 & & $A_{21}(48), A_{11}(24), A_{22}(11)$ \\
\hline & & 1459 & $A_{21}(155), A_{11}(29)$ \\
\hline \multirow[t]{4}{*}{$1458 \mathrm{msh}$} & 1459 & & $A_{12}(71), A_{11}(19)$ \\
\hline & & 1459 & $A_{22}(71), A_{21}(14)$ \\
\hline & & 1459 & $A_{12}(66), A_{11}(19)$ \\
\hline & 1460 & & $A_{22}(75), A_{21}(15)$ \\
\hline
\end{tabular}

$664 \mathrm{~cm}^{-1}$ and $699 \mathrm{~cm}^{-1}$ for the ${ }_{\mathrm{H}} S_{\mathrm{HC}} S_{\mathrm{HC}} S_{\mathrm{H}}$ conformer, and at $666 \mathrm{~cm}^{-1}$ and $689 \mathrm{~cm}^{-1}$ for the ${ }_{\mathrm{H}} S_{\mathrm{CH}} S_{\mathrm{HC}} S_{\mathrm{H}}$ form. The higher frequency in each conformer is assigned to the strong observed band at $688 \mathrm{~cm}^{-1}$. Since no bands were observed close to $666 \mathrm{~cm}^{-1}$ the other two calculated frequencies are left unassigned. The two observed bands at 563 
$\mathrm{cm}^{-1}$ and $635 \mathrm{~cm}^{-1}$, and the identification of the ${ }_{\mathrm{H}} S_{\mathrm{HH}} S_{\mathrm{H}}$ and ${ }_{\mathrm{H}} S_{\mathrm{HH}} S_{\mathrm{H}}$ conformers in isotactic 2,4-dichloropentane, encouraged us to calculate the vibrational frequencies for the ${ }_{\mathrm{H}} S_{\mathrm{HH}} S_{\mathrm{HH}} S_{\mathrm{H}}$ and ${ }_{\mathrm{H}} S_{\mathrm{HH}} S_{\mathrm{HH}} S_{\mathrm{H}}$ conformations of isotactic 2,4,6-trichloroheptane. As seen from Table IV, the ${ }_{\mathrm{H}} S_{\mathrm{HH}} S_{\mathrm{HH}} S_{\mathrm{H}}$ conformer has predicted bands at $565 \mathrm{~cm}^{-1}$ and $628 \mathrm{~cm}^{-1}$, in reasonable agreement with the observed bands at $563 \mathrm{~cm}^{-1}$ and $635 \mathrm{~cm}^{-1}$. We think it likely that this conformer is present at room temperature. A calculated band at $568 \mathrm{~cm}^{-1}$ for the ${ }_{\mathrm{H}} S_{\mathrm{HH}} S_{\mathrm{HH}} S_{\mathrm{H}}$ conformer may also be consistent with the presence of some of this isomer.

The assignment of the observed $\mathrm{C}-\mathrm{Cl}$ stretching vibrations in the heterotactic stereoisomer is not complicated. Two rotational isomers are predicted to be present, ${ }_{\mathrm{H}} S_{\mathrm{HH}} S_{\mathrm{HC}} S_{\mathrm{H}}$ and ${ }_{\mathrm{H}} S_{\mathrm{HH}} S_{\mathrm{CH}} S_{\mathrm{H}}$. In the first conformer there are two adjacent syndiotactic ${ }_{\mathrm{H}} S_{\mathrm{H}} \mathrm{C}-\mathrm{Cl}$ stretching modes which interact and split, and one ${ }_{\mathrm{C}} S_{\mathrm{H}}$ mode associated with the isotactically placed chlorine atom. The observed bands at $610 \mathrm{~cm}^{-1}, 628 \mathrm{~cm}^{-1}$, and $688 \mathrm{~cm}^{-1}$ are assigned to the predicted bands at $607 \mathrm{~cm}^{-1}, 626 \mathrm{~cm}^{-1}$, and $680 \mathrm{~cm}^{-1}$, respectively. In the ${ }_{H} S_{\mathrm{HH}} S_{\mathrm{CH}} S_{\mathrm{H}}$ conformer the two ${ }_{\mathrm{H}} S_{\mathrm{H}}$ modes are not adjacent and do not interact to produce appreciable splitting. These vibrations, calculated at 611 $\mathrm{cm}^{-1}$ and $616 \mathrm{~cm}^{-1}$, are assigned to observed bands at $610 \mathrm{~cm}^{-1}$ and $620 \mathrm{~cm}^{-1}$. The ${ }_{\mathrm{H}} S_{\mathrm{C}}$ vibration which is predicted at $689 \mathrm{~cm}^{-1}$ is assigned to the observed band at 688 $\mathrm{cm}^{-1}$. The observed spectra in the region from $425 \mathrm{~cm}^{-1}$ to $600 \mathrm{~cm}^{-1}$ are also well accounted for in terms of these two conformations of the heterotactic isomer. Higher frequency vibrations agree well with predicted frequencies, but are found to be less sensitive to conformation than are the vibrations which occur below $700 \mathrm{~cm}^{-1}$.

\section{DISCUSSION}

The results of normal coordinate calculations of the vibrational modes of 2,4-dichloropentane and 2,4,6-trichloroheptane are reported. The validity of the general valence force field for secondary chlorides used in these calculations $(8)$ is supported by the good agreement obtained between observed and predicted bands.

In order to account for several weak infrared and Raman bands in the spectra of dl- and meso-2,4-dichloropentane and of 2,4,6-trichloroheptane, it is necessary to relax the restrictions to preferred conformations which are dictated by energy calculations on staggered, tetrahedral molecular models. According to Sykora (5), who has searched the continuum of molecular conformations (using a nonstaggered model) for local energy minima, the staggered model is a good approximation for the preferred conformation but fails to predict accurately the relative importance of the higher energy structures. Flory and Williams (12) evaluated statistical weight parameters, from which the conformer populations of the stereoisomers of 2,4-dichloropentane and 2,4,6-trichloroheptane werc determincd. Their results also indicate that previously ignored rotational isomers are present in some abundance at room temperature.

Attempts to interpret the NMR spectra of 2,4-dichloropentane have most often been made in terms of the presence of only the preferred structures. However, Schneider et al. (4) report that the NMR spectra of $d l$-2,4-dichloropentane cannot be adequately explained in terms of the structures predicted by the staggered molecular model. The temperature dependence of the vicinal coupling constants in the meso stereoisomer also suggest the presence of more than one isomer (4). 
Evidence is presented here which strongly indicates that at room temperature the $d l$ isomer of 2,4-dichloropentane contains, in addition to the ${ }_{\mathrm{H}} S_{\mathrm{HH}} S_{\mathrm{H}}$ and ${ }_{\mathrm{H}} S_{\mathrm{CC}} S_{\mathrm{H}}$ forms, measurable amounts of the ${ }_{\mathrm{H}} S_{\mathrm{HH}^{\prime}} S_{\mathrm{H}},{ }_{\mathrm{H}} S_{\mathrm{HC}} S_{\mathrm{H}}$, and ${ }_{\mathrm{H}} S_{\mathrm{CH}} S_{\mathrm{H}}$ conformations. Meso-2,4-dichloropentane exists in the preferred ${ }_{\mathrm{H}} S_{\mathrm{HC}} S_{\mathrm{H}}$ form as well as in the ${ }_{\mathrm{H}} S_{\mathrm{HH}^{\prime}} S_{\mathrm{H}}$ and ${ }_{\mathrm{H}} S_{\mathrm{HH}} S_{\mathrm{H}}$ conformations. Likewise, the interpretation of the spectra of the stereoisomers of 2,4,6-trichloroheptane require the admission of conformations which were previously believed to be sterically disfavored. In particular, in the $\mathrm{C}-\mathrm{Cl}$ stretching region isotactic 2,4,6-trichloroheptane contains absorption bands which can only be interpreted in terms of less favored structures.

Because the force field refined for secondary chlorides $(8)$ gives such good agreement with the observed vibrational spectra, we feel that the present results provide compelling evidence for the existence of conformers other than the most preferred. Since many of these had previously been rejected on the basis of steric considerations, it is clear that a more realistic analysis of their exact structures [probably along the lines proposed by Sykora (5)] is necessary.

\section{ACKNOWLEDGMENT}

This research was supported by National Science Foundation Grant GP-27717.

ReCEIVED: June 27, 1973

\section{REFERENCES}

1. T. Shimanouchi and M. Tasumi, Spectrochim. Acta 17, 755 (1961).

2. D. Doskočilová and B. Schneider, Coll. Czech. Chem. Commun. 29, 2290 (1964).

3. C. G. Opaskar and S. KRIMM, Spectrochim. Acta 23A, 2261 (1967).

4. B. Schneider, J. Štokr, D. Doskočllová, S. SÝkora, J. Jakeš, and M. Kolínskí, J, Polymer Sci. 22C, 1073 (1969).

5. S. SÝkora, Coll. Czech. Chem. Commun. 33, 3514 (1968).

6. T. Shimanouchi, M. Tasumi, and Y. Abe, Makromol. Chem. 86, 43 (1965).

7. W. H. Moore, J. H. C. Ching, A. V. R. Warrier, and S. KrimM, Spectrochim. Acta 29A, 1847 (1973).

8. W. H. Moore ANd S. Krimm, Spectrochim. Acta 29A, 2025 (1973).

9. S. KRimM, Pure Applied Chem. 16, 369 (1968).

10. W. H. Moore and S. KrimM, to be published.

11. D. Doskočilová, J. Štokr, B. Schneider, H. Pivová, M. Kolínsky, M. Petránek, and D. Lím, J. Polymer Sci. 16, 215 (1967).

12. P. J. Flory AND A. D. Wilitiams, J. Am. Chem. Soc. 91, 3118 (1969). 